\title{
Capturing Correlation Effects on Photoionization Dynamics
}

\author{
Moitra, Torsha; Coriani, Sonia; Decleva, Piero
}

Published in:

Journal of Chemical Theory and Computation

Link to article, DOI:

10.1021/acs.jctc. $1 \mathrm{c} 00303$

Publication date:

2021

Document Version

Publisher's PDF, also known as Version of record

Link back to DTU Orbit

Citation (APA):

Moitra, T., Coriani, S., \& Decleva, P. (2021). Capturing Correlation Effects on Photoionization Dynamics. Journal of Chemical Theory and Computation, 17(8), 5064-5079. https://doi.org/10.1021/acs.jctc.1c00303

\section{General rights}

Copyright and moral rights for the publications made accessible in the public portal are retained by the authors and/or other copyright owners and it is a condition of accessing publications that users recognise and abide by the legal requirements associated with these rights.

- Users may download and print one copy of any publication from the public portal for the purpose of private study or research.

- You may not further distribute the material or use it for any profit-making activity or commercial gain

- You may freely distribute the URL identifying the publication in the public portal

If you believe that this document breaches copyright please contact us providing details, and we will remove access to the work immediately and investigate your claim 


\title{
Capturing Correlation Effects on Photoionization Dynamics
}

\author{
Torsha Moitra, Sonia Coriani,* and Piero Decleva*
}

Cite This: J. Chem. Theory Comput. 2021, 17, 5064-5079

Read Online

ABSTRACT: A highly correlated combination of the equation-ofmotion coupled cluster (EOM-CC) Dyson orbital and the multicentric B-spline time-dependent density functional theory (TDDFT)-based approach is proposed and implemented within the single-channel approximation to describe molecular photoionization processes. The twofold objective of the approach is to capture interchannel coupling effects, missing in the B-spline DFT treatment, and to explore the response of Dyson orbitals to strong correlation effects and its influence on the photoionization observables. We validate our scheme by computing partial cross

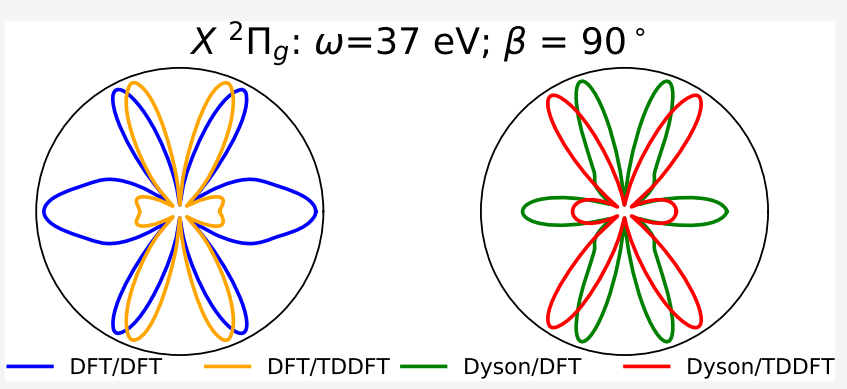
sections, branching ratios, asymmetry parameters, and molecular frame photoelectron angular distributions of simple molecules. Finally, the method has been applied to the study of photoelectron spectra of the $\mathrm{Ni}\left(\mathrm{C}_{3} \mathrm{H}_{5}\right)_{2}$ molecule, where giant correlation effects completely destroy the Koopmans picture.

\section{INTRODUCTION}

The theoretical modeling of photoionization processes is a challenging field of quantum chemistry. It is the process of ejection of an electron to the continuum, and thus, acts as a fingerprint of the target molecule. The description of such processes requires treatment of both the bound target molecule and the outgoing electron at an equal footing. Even though there exist several accurate and robust methodologies for describing the bound state of the system, the same is not true for continuum electrons. In the case of the latter, the complication arises due to the oscillating behavior of the photoelectron wave function and the loss of the usual simple variational principle. Additionally, the basis set should be rich enough to sufficiently emulate the spatial regions far away from the molecule, as the continuum wavefunctions do not decay exponentially at large distances.

An old and popular strategy is to avoid the explicit description of the continuum orbital using the Stieltjes imaging and the equivalent Padé approximant formalisms. ${ }^{1-10}$ These methods are based on the reconstruction of the cross sections from purely bound pseudo spectra. However, it is not possible to obtain individual channel cross sections and asymmetry parameters due to the lack of proper asymptotic boundary conditions, and the energy resolution is relatively modest. Still, these methods have proven quite successful when used in conjunction with highly correlated electronic structure methods such as linear response and equation-of-motion coupled cluster, and algebraic diagrammatic construction. ${ }^{5-8,11,12}$

On the other hand, various approaches have been developed to account for the true nature of the continuum electrons. A rather simple approximation is to describe the outgoing electron by analytical plane waves and Coulomb waves, or their orthogonalized variants. ${ }^{13-15}$ However, these are shown to be not very accurate for studying a wide range of photon energies and specifically fail to reproduce spectral signatures associated with structured continua, like shape resonances and Cooper minima. ${ }^{14,16}$ Alternatively, the continuum orbitals can be determined by stationary (variational) conditions, which can be formulated in technically different, but practically equivalent approaches like the R-matrix, ${ }^{17,18}$ Schwinger variational, ${ }^{19,20}$ complex Kohn, ${ }^{21}$ least-squares or Galerkin methods. ${ }^{22,23}$

A major obstacle in the precise interpretation of photoelectron spectroscopic signals is the need to properly account for correlation effects. These can be separated into two broad categories. The first involves correlation within the bound states, initial and final (sometimes further divided into initial state configuration interaction (ISCI) and final state configuration interaction (FSCI)) and is taken care of using highly correlated wavefunction-based methods, like configuration interaction (CI) ${ }^{24-26}$ perturbation theory, ${ }^{25}$ coupled cluster, ${ }^{13-16}$ and algebraic diagrammatic construction. ${ }^{27}$ The second category involves interaction among different channels, also called interchannel coupling (IC). It can be widely divided into interactions between the electronic continua, the pure openchannel states, and interaction between an electronic continuum and a discrete excitation or a closed channel, which gives rise to autoionization resonances, and describes also the correlation between the bound electrons and the continuum, although it is

Received: March 28, 2021

Published: July 13, 2021 
difficult to draw unambiguous boundaries. In general, the interchannel coupling can be considered as the configuration interaction in the continuum (also known as the final continuum state configuration interaction, FCSCI) and is most complicated to capture, as it requires a general "close-coupling" treatment (see below). ${ }^{28}$ The bound-state correlation, on the other hand, can be well described by quantum chemistry approaches, and then, rather straightforwardly included via Dyson orbitals ${ }^{29,30}$ coupled to an accurate single-particle continuum, according to a single-channel formalism. ${ }^{13-16,31,32}$ This is generally a good approximation, except for the near-threshold region, and far from autoionization resonances. Nevertheless, some interchannel coupling effects are sometimes important, especially when a weak channel is coupled to a much stronger one, with some intensity transfer taking place. At the lowest order, interchannel coupling is often rather well accounted for by the timedependent density functional (TDDFT) approach, that is, the density functional theory analog of $a b$ initio random-phase approximation (RPA), which includes coupling between singly excited configurations.

In this paper, we present a hybrid approach, named Dyson/ TDDFT, coupling an accurate Dyson treatment of the boundstate correlation with the TDDFT continuum, generalizing and improving upon the Dyson-DFT scheme previously presented. ${ }^{16}$ The results are reported for the photoionization observables, namely, partial cross sections $(\sigma)$, branching ratios, asymmetry parameters $(\beta)$, and molecular frame photoelectron angular distributions (MFPADs).

Preliminary results for the argon atom were presented in ref 16. Here, we will further validate the formalism by studying the outer-valence ionization dynamics of simple closed-shell molecules $\left(\mathrm{H}_{2} \mathrm{~S}, \mathrm{CS}_{2}, \mathrm{C}_{3} \mathrm{O}_{2}\right.$, and $\left.\mathrm{C}_{3} \mathrm{~S}_{2}\right)$. With reference to these molecules, we establish a link between the spectral signature and the nature of the bound orbital undergoing ionization. Furthermore, we attempt at identifying the particular scenarios where incorporation of correlation plays a key role. Finally, we examine the photoelectron spectrum of the controversial $\mathrm{Ni}\left(\mathrm{C}_{3} \mathrm{H}_{5}\right)_{2}$ complex, the interpretation of which is not yet settled. It is a famous system where giant correlation/ relaxation effects are active, highlighting the dual effects of bound-state correlation and interchannel coupling, thereby justifying the widespread applicability and accuracy of our scheme. An additional comparison with pure DFT/TDDFT results highlights the very different behavior of DFT and $a b$ initio approaches.

\section{THEORETICAL APPROACH}

2.1. Overview. The theoretically relevant quantity for describing photoelectron spectroscopic observables is the photoelectron matrix element

$$
\vec{D}^{i f}=\left\langle\Psi_{i}^{N}|\vec{\mu}| \Phi_{f}\right\rangle
$$

connecting the initial $N$-electron state $\left(\Psi_{i}^{N}\right)$ and the final composite system described by the wavefunction $\Phi_{f} ; \vec{\mu}$ is the electric dipole moment. In principle, the final continuum can be described by the so-called "close coupling" form, which is equivalent to the configuration interaction (CI) approach in the continuum for the final composite system

$$
\Phi_{f} \equiv \Phi_{E I \alpha}=\mathcal{A} \sum_{J}\left(\Psi_{J}^{N-1} \phi_{E J I \alpha}\right)+\sum_{K} \Phi_{K}^{N} C_{E K I}
$$

The first term of eq 2 comprises all possible antisymmetrized products of $(N-1)$-electron final bound states, $\Psi_{J}^{N-1}$, accessible at energy $E$, coupled to continuum wavefunctions, $\phi_{E J I \alpha}$. The index $J$ counts the different ionic (target) states included in the expansion, which give rise to an equivalent number of final independent solutions, indexed by $I ; \alpha$ enumerates the independent continuum partial waves. The second term is a linear combination of $N$-electron localized basis functions, $\Phi_{K}^{N}$, with coefficients $C_{E K I}$, accounting for both autoionizing states and short-range correlation effects. The $a b$ initio computation using the complete expression is computationally very demanding and is usually restricted to small systems and low energies. ${ }^{33-36}$ Several approximate formulations have been developed, differing both in the level of truncation of the closecoupling expression and in the treatment of the continuum orbitals $\phi_{E I J \alpha^{*}}{ }^{20,37-40}$

We consider the single-channel approximation, where the second term of eq 2 is neglected and only one bound ionized state is considered at a particular energy. Hence, the expression for the final state becomes

$$
\Phi_{E I \alpha}=\mathcal{A}\left(\Psi_{I}^{N-1} \phi_{E I \alpha}\right)
$$

The simplified expression, eq 3, lacks coupling between open channels and correlation between the bound state and the continuum. However, these effects are weak, far from the ionization threshold and in the absence of autoionizing resonances. Interestingly, within the single-channel approximation, the many-particle photoelectron matrix element reduces to single-particle matrix elements ${ }^{16,24,41}$

$$
\begin{aligned}
& D_{\gamma}^{i f}=\left\langle\Psi_{i}^{N}\left|\mu_{\gamma}\right| \Phi_{f}\right\rangle=\left\langle\phi_{i f}^{d}\left|\mu_{\gamma}\right| \phi_{E I \alpha}\right\rangle+\left\langle\eta_{i f, \gamma}^{d} \mid \phi_{E I \alpha}\right\rangle \\
& \phi_{i f}^{d}=\left\langle\Psi_{I}^{N-1}|\hat{\psi}(x)| \Psi_{i}^{N}\right\rangle, \quad \eta_{i f, \gamma}^{d}=\left\langle\Psi_{I}^{N-1}\left|\mu_{\gamma} \hat{\psi}(x)\right| \Psi_{i}^{N}\right\rangle
\end{aligned}
$$

where index $\gamma$ labels a specific Cartesian component of the dipole operator, and $\phi_{i f}^{d}$ and $\eta_{i f, \gamma}^{d}$ are the Dyson orbital and the conjugate Dyson orbital, respectively. Note the use, in eq 5, of the field annihilation operator $\hat{\psi}(x)$, which can be expanded in terms of a complete basis as

$$
\hat{\psi}(x)=\sum_{q} \phi_{q}(x) a_{q}
$$

where $a_{q}$ is the annihilation operator. These Dyson orbitals can be expressed entirely in terms of the bound-state wavefunction and embody all correlations necessary for their description.

The first Dyson term in eq 4 arises from a dipole transition of initial orbitals to the continuum, multiplied by the overlap of the passive ones. The second term, conjugate, arises from a dipole transition within the bound orbitals, and an overlap of the remaining one with the continuum..$^{16,24,41}$ It appears because of nonorthogonality of the continuum to the initial bound eigenstate and is zero if all bound orbitals in the initial state are orthogonal to it. Moreover, its relative intensity with respect to the Dyson term decreases with increasing electron energy. Apart from special cases, its contribution is generally very small and is neglected. A well-established formalism to obtain the photoelectron matrix element uses single determinants built from the occupied orbitals at the ground state, and variationally computes the continuum. This is known as the frozen HartreeFock, or static exchange (SE) method. Alternatively, an equivalent formulation in the DFT regime, namely the static exchange DFT (SE-DFT) is obtained employing both bound 
and continuum orbitals as eigenstates of the Kohn-Sham DFT Hamiltonian, determined from the ground-state density. ${ }^{42,43}$ At this level, employing a common set of orthonormal orbitals (bound + continuum), the continuum is orthogonal to all initial orbitals and the overlapping term is zero. The same is true at the SCI or TDDFT levels. When correlation is included in the bound states, or relaxation is allowed, orthogonality breaks down. In the case of very strong orbital relaxation, like in the case of double-core holes, the conjugate term may actually become comparable with the direct one. ${ }^{44}$ When employing a correlated ionic state, the SE-DFT continuum is no longer variational, although combining it with the Dyson orbital is still a good approximation, as previously shown. ${ }^{16,31,32,45}$ We refer to it as Dyson/DFT formulation.

The simplest method that includes interchannel coupling effects (correlation between the continuum channels), while still treating the bound states as a single determinant, is the singly excited CI (CIS) approach or the Tamm-Dancoff approximation (TDA). An additional correlation may be included via the random-phase approximation (RPA), in the linear response formalism, still conserving the essential CIS structure of excitation. Similarly, the equivalent formulation within DFT is the TDDFT approach. It is interesting that TDDFT (or RPA) can be seen in two equivalent, but physically quite distinct, ways. The one most familiar to chemists is a basis set formulation, known as the Casida approach, ${ }^{46}$ which clearly shows the correspondence to CIS. The second is from the response viewpoint, which describes the screening of the external exciting field due to the response of the electron cloud. ${ }^{47-49}$ The fundamental equations governing the linear response are

$$
\delta \rho=\chi V^{\mathrm{SCF}}, \quad \delta V=K \delta \rho, \quad V^{\mathrm{SCF}}=\mu^{\mathrm{ext}}+\delta V
$$

where $\delta \rho$ is the first-order change in the electron density induced by the field, $\chi$ is the electric susceptibility, $\delta V$ is the first-order change in the potential induced by a density change $\delta \rho$, and $K$ is the linear kernel relating the two quantities. The final full potential $\left(V^{\mathrm{SCF}}\right)$ is the sum of the external perturbing potential $\left(\mu^{\text {ext }}\right)$ and the response potential $(\delta V)$. It is known that the effect-i.e., interchannel coupling in the first interpretation, and screening in the second-may be quite strong, especially close to the threshold. While in the first viewpoint, the coupling of this continuum description with a single-channel formulation may appear strange, it is more natural in the latter. In the response framework, the effect of electron polarization gives rise to an effective potential, which is the sum of the external dipole field and the electronic response potential. The evaluation of the photoelectron matrix element then boils down to replacing the pure dipole field in eq 4 by the effective potential $V^{S C F}$

$$
D_{\gamma}^{i f}=\left\langle\Psi_{i}^{N}\left|V_{\gamma}^{\mathrm{SCF}}\right| \Phi_{f}\right\rangle
$$

From this expression, introducing a Dyson/TDDFT approach comes naturally, yielding

$$
D_{\gamma}^{i f}=\left\langle\phi_{i f}^{d}\left|V_{\gamma}^{\mathrm{SCF}}\right| \phi_{E I \alpha}\right\rangle
$$

We have implemented and discussed the Dyson/TDDFT formulation, eq 8 , in this article. The length gauge has been used throughout. The details of the algorithm are described in the following subsections.

2.2. Linear Combination of Atomic Orbital (LCAO) BSpline TDDFT Continuum. Solving for the continuum orbital within the TDDFT approach is quite involved. As the TDDFT formalism can be considered an extension to the Kohn-Sham DFT (KS-DFT), we start from the latter as input for computing the TDDFT continuum orbitals. At the KS-DFT stage, the continuum orbitals $\left(\phi_{\epsilon l m}\right)$ are computed by solving the Schrödinger equation in the angular momentum representation, with $l$ and $m$ as usual angular momentum quantum numbers

$$
H_{\mathrm{KS}} \phi_{\mathrm{elm}}=E \phi_{\mathrm{elm}}
$$

where

$$
H_{\mathrm{KS}}=-\frac{1}{2} \Delta-\sum_{N} \frac{Z_{N}}{\left|\bar{r}-\bar{R}_{N}\right|}+\int \frac{\rho\left(\bar{r}^{\prime}\right) \mathrm{d} \bar{r}^{\prime}}{\left|\bar{r}-\bar{r}^{\prime}\right|}+V_{\mathrm{XC}}[\rho(\bar{r})]
$$

Here, $H_{\mathrm{KS}}$ is the KS Hamiltonian and $\rho(\bar{r})$ is the ground-state electron density. For $V_{\mathrm{XC}}$, we have here chosen the LB94 exchange-correlation potential due to its proper description of the asymptotic Coulomb behavior. ${ }^{43}$ The continuum KS wavefunctions are then obtained as eigenvectors with minimum modulus eigenvalue of the energy-dependent matrix $\mathbf{A}^{\dagger} \mathbf{A}$

$$
\mathbf{A}^{\dagger} \mathbf{A}(E) c=a c, \quad \mathbf{A}(E)=\mathbf{H}-E \mathbf{S}
$$

where $\mathbf{H}$ and $\mathbf{S}$ are the Hamiltonian and overlap matrices, respectively, $E$ is photoelectron kinetic energy, $c$ is the eigenvector, and $a$ is the minimum modulus eigenvalue. ${ }^{50}$

From eqs 6, one can obtain an equation for $V^{\mathrm{SCF}}$

$$
\begin{aligned}
V^{\mathrm{SCF}} & =\mu^{\mathrm{ext}}+K \delta \rho \\
& =\mu^{\mathrm{ext}}+K \chi V^{\mathrm{SCF}}
\end{aligned}
$$

We then solve directly for the response potential $\left(V^{S \mathrm{CF}}\right)$ as the prime dynamical variable, using a noniterative numerical algorithm. ${ }^{50}$ The kernel $K$ is the sum of the Coulomb potential and the linearized exchange-correlation response

$$
K\left(\bar{r}, \bar{r}^{\prime}\right)=\frac{1}{\left|\bar{r}-\bar{r}^{\prime}\right|}+\delta\left(\bar{r}-\bar{r}^{\prime}\right) \frac{\delta V_{\mathrm{XC}}}{\delta \rho}
$$

By representing $V^{\mathrm{SCF}}$ as well as the operators $K$ and $\chi$ in the Bspline basis, the integrodifferential equation, eq 12 , is converted into a linear algebraic one

$$
(\mathbf{K} \chi-\mathbf{1}) \mathbf{V}^{\mathrm{SCF}}=-\boldsymbol{\mu}^{\mathrm{ext}}
$$

The computationally expensive part is the calculation of the KS (noninteracting) linear susceptibility, which is energy-dependent and is computed for each photon energy by solving the inhomogeneous first-order perturbative equations. The completeness of the basis ensures convergent solutions, and the full details are available in ref 50 .

As anticipated, we employ a basis set expansion, with primitives constructed as products of radial B-splines $\left(B_{j}(\bar{r})\right)$ and real spherical harmonics $\left(Y_{l m}(\theta, \phi)\right)$ located on different centers, in the spirit of a linear combination of atomic orbitals (LCAO)

$$
\chi_{j l m}(\bar{r}, \theta, \phi)=\frac{1}{r} B_{j}(\bar{r}) Y_{l m}(\theta, \phi)
$$

The B-splines are defined on a given interval, say $\left[0, R_{\max }\right]$, divided into subintervals by a nondecreasing sequence of points (knots). Each B-spline is made of different polynomial pieces over $n$ successive knots ( $n$ is the fixed order of the polynomials) joined with a high degree of continuity between adjacent subintervals, and is zero outside, so that exactly $n$ splines overlap on each subinterval, and linear independence is optimally 
assured. The B-splines can approximate any smooth function in the given interval with arbitrary accuracy by decreasing the knot spacing and increasing the polynomial order. ${ }^{51}$ They enjoy the flexibility of finite difference methods on a grid, with the ease of manipulation of basis sets via linear algebra algorithms. In our implementation, a long-range expansion is located on the origin of the molecule (one center, OCE), supplemented with short expansions around the nuclei, to account for Coulomb cusps. The OCE expansion defines a sphere large enough that bound states are negligible outside, and the oscillating continuum has reached the asymptotic limit and can be fitted to the known analytic solutions (coulomb functions for photoionization). ${ }^{42}$

2.3. EOM-CCSD Dyson Orbitals. The one-electron Dyson orbital is defined as the overlap between the bound initial $\mathrm{N}$ electron and final $(N-1)$-electron states, given by

$$
\begin{aligned}
\phi_{i f}^{d} & =\sqrt{N} \int \Psi_{i}^{N}\left(x_{1}, x_{2}, \ldots, x_{N}\right) \Psi_{f}^{N-1}\left(x_{2}, \ldots, x_{N}\right) \mathrm{d} x_{2} \ldots \mathrm{d} x_{N} \\
& \equiv \sum_{p} \gamma_{p} \phi_{p}
\end{aligned}
$$

It can be further reduced to a linear combination of ground-state molecular spin-orbitals $\left(\phi_{p}\right)$, with combination coefficients $\gamma_{p}$. In a single determinant description, considering ionization from the $k$ th molecular orbital, the Dyson orbital reduces to the initial occupied orbital $\phi_{k}$, and the corresponding ionization energy is equal to the negative $k$ th orbital energy, according to Koopmans' theorem $^{52}$

$$
\phi_{i f}^{d}=\phi_{k} \text { and } \gamma_{p}=\delta_{k p}
$$

Here, we use the EOM-CCSD framework, ${ }^{53,54}$ where the bound states are obtained by diagonalizing the matrix representation of the non-Hermitian similarity-transformed Hamiltonian, $\bar{H}=$ $\exp (-T) H \exp (T)$, on the reference and the truncated excitation space

$$
\overline{\mathbf{H}} \mathbf{R}_{m}=E_{m} \mathbf{R}_{m}, \quad \mathbf{L}_{m}^{\dagger} \overline{\mathbf{H}}=\mathbf{L}_{m}^{\dagger} E_{m} \text { and } \mathbf{L}_{m}^{\dagger} \mathbf{R}_{n}=\delta_{m n}
$$

In connection to ionization processes, the final bound $(N-1)$ state wavefunction can be expressed (for CCSD) as

$$
\left|\Psi^{N-1}\right\rangle=R^{\mathrm{IE}}\left|\Psi_{\mathrm{CCSD}}^{N}\right\rangle=R^{\mathrm{IE}} \exp \left(T_{1}+T_{2}\right)\left|\Phi_{0}\right\rangle
$$

where

$$
R^{\mathrm{IE}}=\sum_{i} r_{i} a_{i}+\frac{1}{2} \sum_{a i j} r_{i j}^{a} a_{a}^{\dagger} a_{j} a_{i}
$$

and $\left|\Psi_{\mathrm{CCSD}}^{N}\right\rangle$ and $\left|\Phi_{0}\right\rangle$ are the CCSD ground-state wavefunction and the reference Hartree-Fock Slater determinant for the $N$ electron state, respectively. $T, R^{\mathrm{IE}}$, and $\left(L^{\mathrm{IE}}\right)^{\dagger}$ are excitation operators from a reference determinant. The operator $R^{\mathrm{IE}}$ (and similarly $L^{\mathrm{IE}}$ ) is not electron-conserving, as shown in eq 20. Indices $i$ and $j$ refer to occupied orbitals and the index $a$ to virtual orbitals. In coupled cluster theory, bra (left) and ket (right) states are not each other's adjoint. ${ }^{55}$ As a consequence, the left $\left(\phi^{d, \mathrm{~L}}\right)$ and right $\left(\phi^{d, \mathrm{R}}\right)$ EOM-CCSD Dyson orbitals are different, i.e., their coefficients $\gamma_{p}^{\mathrm{L}} / \gamma_{p}^{\mathrm{R}}$ are distinct. For ionization from the ground state, the $\gamma_{p}$ coefficients are ${ }^{15}$

$$
\begin{aligned}
\gamma_{p}^{\mathrm{L}} & =\left\langle\Psi_{\mathrm{L}}^{N-1}\left|a_{p}\right| \Psi_{\mathrm{R}}^{N}\right\rangle \\
& =\left\langle\mathrm{HF}\left|L^{\mathrm{IE}} \exp \left(-T_{2}-T_{1}\right) a_{p} \exp \left(T_{1}+T_{2}\right)\right| \mathrm{HF}\right\rangle
\end{aligned}
$$

and

$$
\begin{aligned}
\gamma_{p}^{\mathrm{R}} & =\left\langle\Psi_{\mathrm{L}}^{N}\left|a_{p}^{\dagger}\right| \Psi_{\mathrm{R}}^{N-1}\right\rangle \\
& =\left\langle\mathrm{HF}\left|(1+\hat{\Lambda}) \exp \left(-T_{2}-T_{1}\right) a_{p}^{\dagger} R^{\mathrm{IE}} \exp \left(T_{1}+T_{2}\right)\right| \mathrm{HF}\right\rangle
\end{aligned}
$$

A suitable inclusion of the bound-state correlations and even treatment of nonsingle determinant states (for example, satellites) as complex open shells can be obtained by employing the Dyson orbitals.

The squared norm of the Dyson orbital, defined as

$$
R_{F}=\left\|\phi^{d}\right\|^{2}=\sum_{p} \gamma_{p}^{2}
$$

is the spectral strength, also called the pole strength or spectroscopic factor, of the final state $\Psi^{N-1}$. The values of $R_{F}$ are used as the intensity of bands when simulating the photoelectron spectrum. As a known effect of inclusion of correlation, the spectral strengths of the primary ionic states are lowered and, at the same time, additional states, i.e., satellite or shake-up states, which are characterized by further electronic excitations, gain intensity. As the right and left Dyson orbitals are different in EOM-CC, one may ponder how to compute the spectroscopic factor, for instance, whether one should take the square norm of just one of the two Dyson orbitals (left or right), or to use the product of their norms (geometric norm), or a third recipe altogether. ${ }^{56,57}$ Here, we propose to use, as a spectroscopic factor, the dot product of the corresponding left and right Dyson orbital pairs

$$
R_{F}=\sum_{p}\left(\gamma_{p}^{d, \mathrm{~L}} \gamma_{p}^{d, \mathrm{R}}\right)
$$

It is worth noticing, nonetheless, that the differences between the numerical results of the different definitions are almost negligible (see, e.g., Figure S2).

2.4. Photoionization Parameters. The differential partial cross section in atomic units (in the molecular frame) is given by

$$
\frac{\mathrm{d} \sigma}{\mathrm{d} \vec{k}}=4 \pi^{2} \alpha \omega\left|D^{i f}\right|^{2}
$$

where $\alpha$ is the fine structure constant, $\omega$ is the photon energy, and $\vec{k}$ is the momentum of the photoelectron in the molecular frame.

In the case of randomly oriented molecules, following the standard manipulations as adopted by Chandra, ${ }^{58}$ one obtains

$$
\frac{\mathrm{d} \sigma}{\mathrm{d} \vec{k}}=\pi \alpha \omega(-1)^{m_{r}} \sum_{L} A_{L}(k) P_{L}(\cos \theta)
$$

where $m_{r}$ specifies the polarization of light with possible values of $0,+1$, and -1 for linearly polarized (LP), left circularly polarized (LCP), and right circularly polarized (RCP) light, respectively; $\theta$ is the scattering angle between $\vec{k}$ and the laboratory frame defined by the electric field (for LP) or direction of propagation of light (for CP). The coefficients $A_{L}(k)$ are given by 


$$
\begin{aligned}
& A_{L}(k)=(2 L+1)\left(\begin{array}{lll}
1 & 1 & L \\
m_{r} & -m_{r} & 0
\end{array}\right) \sum_{\substack{l m \gamma \\
l^{\prime} m^{\prime} \gamma^{\prime}}}(-1)^{m+\gamma} \\
& \quad \sqrt{(2 l+1)\left(2 l^{\prime}+1\right)} \\
& \times\left(\begin{array}{lll}
l & l^{\prime} & L \\
0 & 0 & 0
\end{array}\right)\left(\begin{array}{lll}
l & l^{\prime} & L \\
-m & m^{\prime} & \left(m-m^{\prime}\right)
\end{array}\right)\left(\begin{array}{lll}
1 & 1 & L \\
\gamma^{\prime} & -\gamma & \left(\gamma-\gamma^{\prime}\right)
\end{array}\right) \\
& \times \frac{1}{2}\left(D_{\epsilon l m, \gamma}^{\mathrm{R}} D_{\epsilon l^{\prime} m^{\prime}, \gamma^{\prime}}^{\mathrm{L}^{*}}+D_{\epsilon l m, \gamma}^{\mathrm{L}} D_{\epsilon l^{\prime} m^{\prime}, \gamma^{\prime}}^{\mathrm{R}^{*}}\right)
\end{aligned}
$$

$P_{L}$ are Legendre polynomials of order $L$, where $L=0,1,2$, and $D_{e l m, \gamma}^{\mathrm{R}}$ and $D_{e l m, \gamma}^{\mathrm{L}}$ indicate specific right and left photoelectron dipole matrix elements in the Cartesian direction $\gamma$. For more details, refer to the Supporting Information of ref 16. Defining

$$
\begin{aligned}
\frac{\sigma}{4 \pi} & =\pi \alpha \omega(-1)^{m_{r}} A_{0} ; \quad D=\frac{A_{1}\left(m_{r}=1\right)}{A_{0}} ; \\
\beta & =\frac{A_{2}\left(m_{r}=0\right)}{A_{0}}
\end{aligned}
$$

where $\sigma$ is the partial cross section, $\beta$ is the asymmetry parameter, and $D$ is the dichroic parameter, allows us to rewrite the final expression for the differential cross section as

$$
\frac{\mathrm{d} \sigma}{\mathrm{d} \vec{k}}=\frac{\sigma}{4 \pi}\left[1+\left(-\frac{1}{2}\right)^{\left|m_{r}\right|} \beta P_{2}(\cos \theta)+m_{r} D P_{1}(\cos \theta)\right]
$$

Equation 29 corroborates that the angular distribution of the photoelectrons only depends on the cross section $\sigma$, asymmetry parameter $\beta$, and the dichroic parameter $D$. We will in the following only consider linearly polarized light, i.e., $m_{r}=0$, so eq 29 boils down to

$$
\frac{\mathrm{d} \sigma}{\mathrm{d} \vec{k}}=\frac{\sigma}{4 \pi}\left[1+\beta P_{2}(\cos \theta)\right]
$$

Besides partial cross-sections and asymmetry parameters, we will also study the branching ratio, defined as the ratio between a specific cross section and the sum of all considered cross sections. Additionally, molecular frame photoelectron angular distributions (MFPADs) are computed as the polar plot of the differential partial cross section, following the general treatment described in, e.g., refs 58, 59.

\section{COMPUTATIONAL DETAILS}

Experimental geometries from the NIST compilation ${ }^{60}$ have been used for $\mathrm{H}_{2} \mathrm{~S}$ and $\mathrm{CS}_{2}$. The geometry of the nickel complex is the same as used in ref 61. They are reported in the Supporting Information file as Cartesian coordinates. The ground-state electronic densities were obtained from the $\mathrm{ADF}$ program package $^{62}$ using the LB94 exchange-correlation potential with the DZP basis set. ${ }^{63}$ Following this step, both the bound and continuum orbitals were then computed in the LCAO B-spline basis, with a suitable choice of parameters, which is given in Table 1. With this choice of parameters, we ensured accurate convergence of the photoionization results.

The ionization energies and corresponding Dyson orbitals were computed at the EOM-CCSD level using Q-Chem. ${ }^{64}$ CCSDR(3) results for the ionization energies of $\mathrm{CS}_{2}$ were obtained using Dalton. A comparison of the photoelectron spectra obtained employing different descriptions of the bound states is shown in Figure S1. Dunning's correlation consistent
Table 1. Parameters Used in the LCAO B-Spline Code ${ }^{a}$

\begin{tabular}{lccccc} 
& & & & \multicolumn{2}{c}{ LCAO descriptor } \\
\cline { 5 - 6 } molecule & $\begin{array}{c}\text { no. of } \\
\text { knots }\end{array}$ & $L_{\max }$ & $R_{\max }$ & \multicolumn{1}{c}{$l_{\max }$} & $r_{\max }$ \\
$\mathrm{H}_{2} \mathrm{~S}$ & 100 & 12 & 25.0 & $1(\mathrm{H})$ & $0.600(\mathrm{H})$ \\
$\mathrm{CS}_{2}$ & 100 & 20 & 25.0 & $2(\mathrm{~S})$ & $1.000(\mathrm{~S})$ \\
$\mathrm{C}_{3} \mathrm{O}_{2}$ & 100 & 18 & 25.0 & $2(\mathrm{C}, \mathrm{O})$ & $0.992(\mathrm{C})$, \\
& 100 & 16 & 25.0 & $2(\mathrm{C}, \mathrm{S})$ & $1.000(\mathrm{C})$, \\
$\mathrm{C}_{3} \mathrm{~S}_{2}$ & 100 & 16 & 25.0 & $2(\mathrm{C}), 1(\mathrm{H})$ & $1.300(\mathrm{C})$, \\
& & & & & $0.700(\mathrm{H})$
\end{tabular}

${ }^{a} L_{\max }$ and $l_{\max }$ are the maximum angular momentum employed in the one-center expansion at the origin and on each off-center atom, respectively. $R_{\max }$ and $r_{\max }$ (both in au) are the maximum radial grid length from the origin and from the off-center atoms, respectively.

basis sets augmented with polarization and diffuse functions (aug-cc-pVXZ) were used for the small molecules ( $\mathrm{X}=\mathrm{T}$ for $\mathrm{H}_{2} \mathrm{~S} ; \mathrm{X}=\mathrm{D}$ for $\mathrm{CS}_{2}, \mathrm{C}_{2} \mathrm{O}_{3}$, and $\left.\mathrm{C}_{2} \mathrm{~S}_{3}\right)$. In the case of the nickel bisallyl complex, the cc-pV $(\mathrm{D}+\mathrm{d}) \mathrm{Z}$ basis set for the $\mathrm{Ni}$ atom and the DZP basis for $\mathrm{C}$ and $\mathrm{H}$ were employed. To reduce the computational cost, we froze the 1s orbital of the six carbon atoms and up to the $2 \mathrm{p}$ orbitals of $\mathrm{Ni}$. Note that we present the results as per conventional symmetry notations, even though in the Q-Chem calculations a non-Mulliken representation is adopted. All remaining calculations involving the continuum have been performed using the Tiresia code. ${ }^{42}$

Throughout the article, we follow the conventional nomenclature of designating the first ionization band as $X$, the second as $A$, the third as $B$, and so on for the outer-valence ionizations. At higher ionization energies, satellite bands and breakdown of the orbital picture appear. We have not considered this region in detail, as very few accurate photoionization parameters are available, and the present correlation level is not generally accurate enough for a quantitative description.

Here, we first validate the Dyson/TDDFT approach over small molecules $\left(\mathrm{H}_{2} \mathrm{~S}, \mathrm{CS}_{2}, \mathrm{C}_{3} \mathrm{O}_{2}\right.$, and $\left.\mathrm{C}_{3} \mathrm{~S}_{2}\right)$ by comparing the performance of the Hartree-Fock molecular orbitals, the KSDFT molecular orbitals, and the EOM-CCSD Dyson orbitals for the bound-state description, in conjunction with the corresponding LCAO B-spline DFT and TDDFT continuum. To avoid discussion of similar results and due to the lack of experimental profiles for $\mathrm{C}_{3} \mathrm{O}_{2}$ and $\mathrm{C}_{3} \mathrm{~S}_{2}$, they are presented in the Supporting information. Second, we test our approach on the highly correlated spectrum of the $\mathrm{Ni}\left(\mathrm{C}_{3} \mathrm{H}_{5}\right)_{2}$ complex, whose interpretation is still controversial.

As anticipated, results are reported for ionization energies, spectroscopic factors $R_{F}$ branching ratios, partial cross sections $\sigma$, asymmetry parameters $\beta$, and MFPADs.

\section{RESULTS AND DISCUSSION}

4.1. Hydrogen Sulfide. The outer-valence photoionization spectrum of $\mathrm{H}_{2} \mathrm{~S}$ has been reported by several authors, and we here specifically refer to the experimental results by Baltzer et al. ${ }^{65}$ Our results for the ionization energies and pole strengths are collected in Table 2. The photoelectron spectrum is reproduced in Figure S1. It comprises three well-separated bands due to the ionization from the $2 b_{1}, 5 a_{1}$, and $2 b_{2}$ molecular orbitals. All of the three (right) EOM-CCSD Dyson orbitals (shown as insets to Figure 1) have significant contributions from the central sulfur atom. The $X$ band is purely due to ionization 
Table 2. $\mathrm{H}_{2} \mathrm{~S}$ : Hartree-Fock Ionization Energies According to Koopmans' Theorem, LB94 Ground-State KS Eigenvalues, and EOM-CCSD Ionization Energies Versus the

Experiment $^{a}$

$\begin{array}{cccccc}\text { state } & \text { HF } & \text { LCAO KS } & \text { EOM-CCSD } & R_{F} & \text { exp. } \\ X^{2} B_{1} & 10.48 & 10.85 & 10.42 & 0.921 & 10.46 \\ A^{2} A_{1} & 13.66 & 13.72 & 13.43 & 0.913 & 13.45 \\ B^{2} B_{2} & 16.05 & 15.57 & 15.69 & 0.914 & 15.55\end{array}$

${ }^{a}$ All values are in $\mathrm{eV}$. The EOM-CCSD pole strengths are reported in the second last column. Experimental results are taken from ref 68 .

from the $3 p_{x}$ lone pair electron over the $S$ atom, thereby having the largest atomic contribution among the three. The Dyson orbital, corresponding to the $A$ band, is quite similar to the $5 a_{1}$ molecular orbital. Both $A$ and $B$ bands are strongly bonding, with mainly $\mathrm{S}-\mathrm{H} \sigma$ bond nature, with the $B$ band showing a broader vibrational envelope. As the transitions occur from orbitals having some S 3p character, it is anticipated that their crosssections and asymmetry parameters will show the characteristic behavior of the atomic component. The ionization from $3 p$ orbitals of the third-period elements exhibits Cooper minimum. For sulfur, this leads to a pronounced dip in the asymmetry parameter at around $40 \mathrm{eV}$. Remarkably, the dip is the largest in the case of the $X$ band, corroborating the fact that it has the maximum sulfur $3 p$ contribution. In general, the orbital composition is reflected in the photoionization parameters through the dipole matrix elements with the continuum. In the molecular case, the loss of symmetry results in many partial waves contributing, so it becomes difficult to analyze contributions in detail. However, typical atomic features survive in the molecular cross section. They are very useful to identify atomic contributions in the initial orbitals, and even quantify trends, like the participation of a specific AO to different molecular orbitals, or to analogous orbitals in different compounds. The depth of a Cooper minimum is one example, as also seen in the following $\mathrm{CS}_{2}$ case; other examples are delayed onset characteristics of $\mathrm{d}$ and $\mathrm{f}$ AOs, especially in the heavier atoms, atomic shape resonances like $d \rightarrow f$ excitations, and characteristic autoionization resonances. Other features are characteristics of bonds, like shape resonances that can be associated with excitations into $\sigma^{*}$ antibonding orbitals, or diffraction features, which are very sensitive to bond length and molecular geometry. ${ }^{66,67}$

The branching ratio profiles show some features near the threshold and then become flat above $60 \mathrm{eV}$. As the Dyson orbitals are very similar to the corresponding Hartree-Fock and DFT molecular orbitals, no noticeable difference is observed upon variation in the bound-state description. On the other hand, the spectral feature is sensitive to the use of either DFT or TDDFT continuum orbitals. A maximum appears in all branching ratio profiles of the $\mathrm{B}$ band using the TDDFT continuum at around $33-35 \mathrm{eV}$, which is not captured by the DFT approach. Insets zooming into this region are shown in Figure 1. Also, the discrepancy is clearly manifested in the asymmetry parameter profiles. The DFT continuum consistently underestimates the photon energy corresponding to the Cooper minimum by $6-8 \mathrm{eV}$.
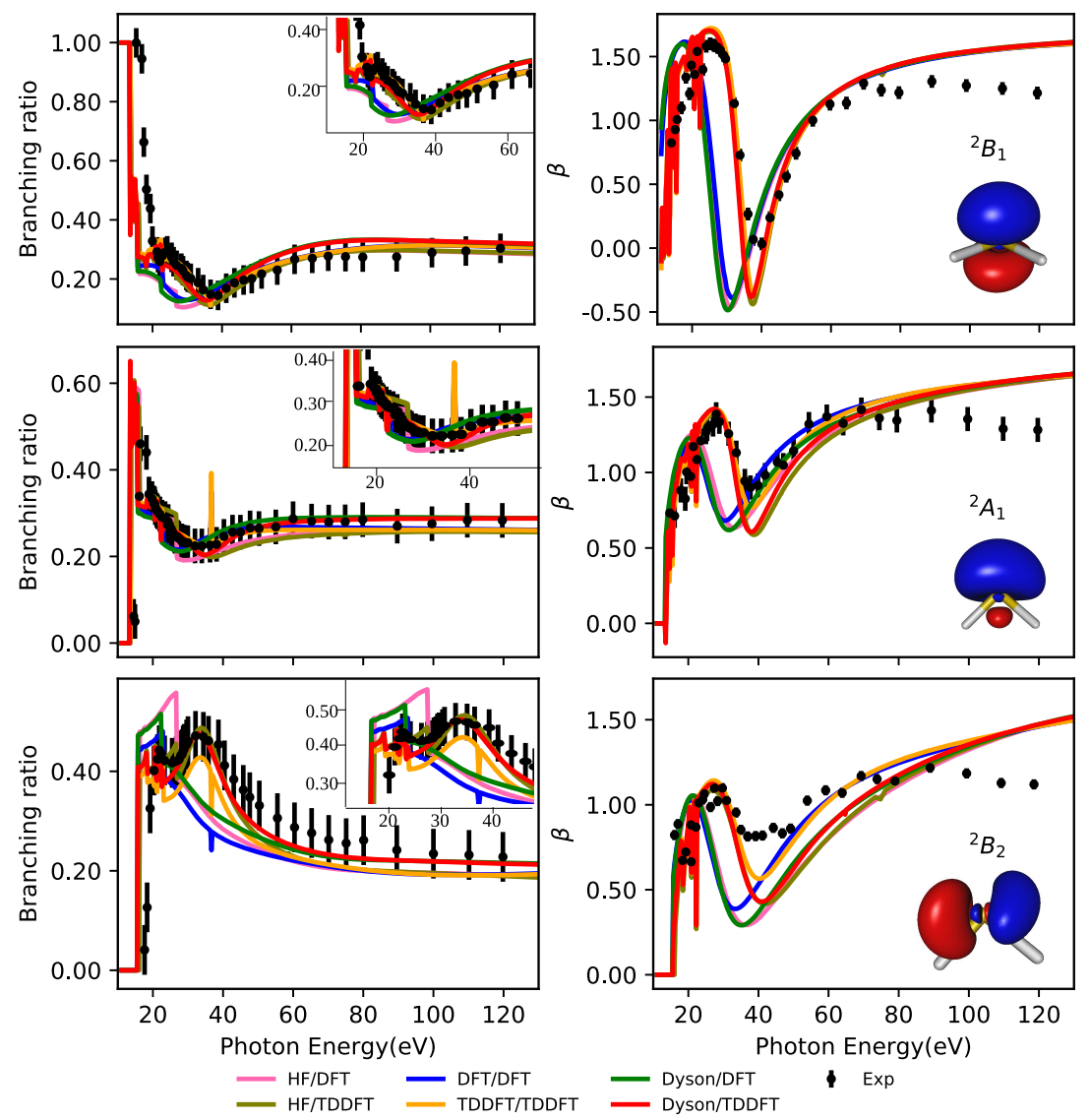

Figure 1. $\mathrm{H}_{2} \mathrm{~S}$ : Branching ratio and asymmetry parameter for the three outer-valence ionizations. Experimental results are taken from ref 65 . Zoomedin branching ratio and right EOM-CCSD Dyson orbitals are plotted as insets. 

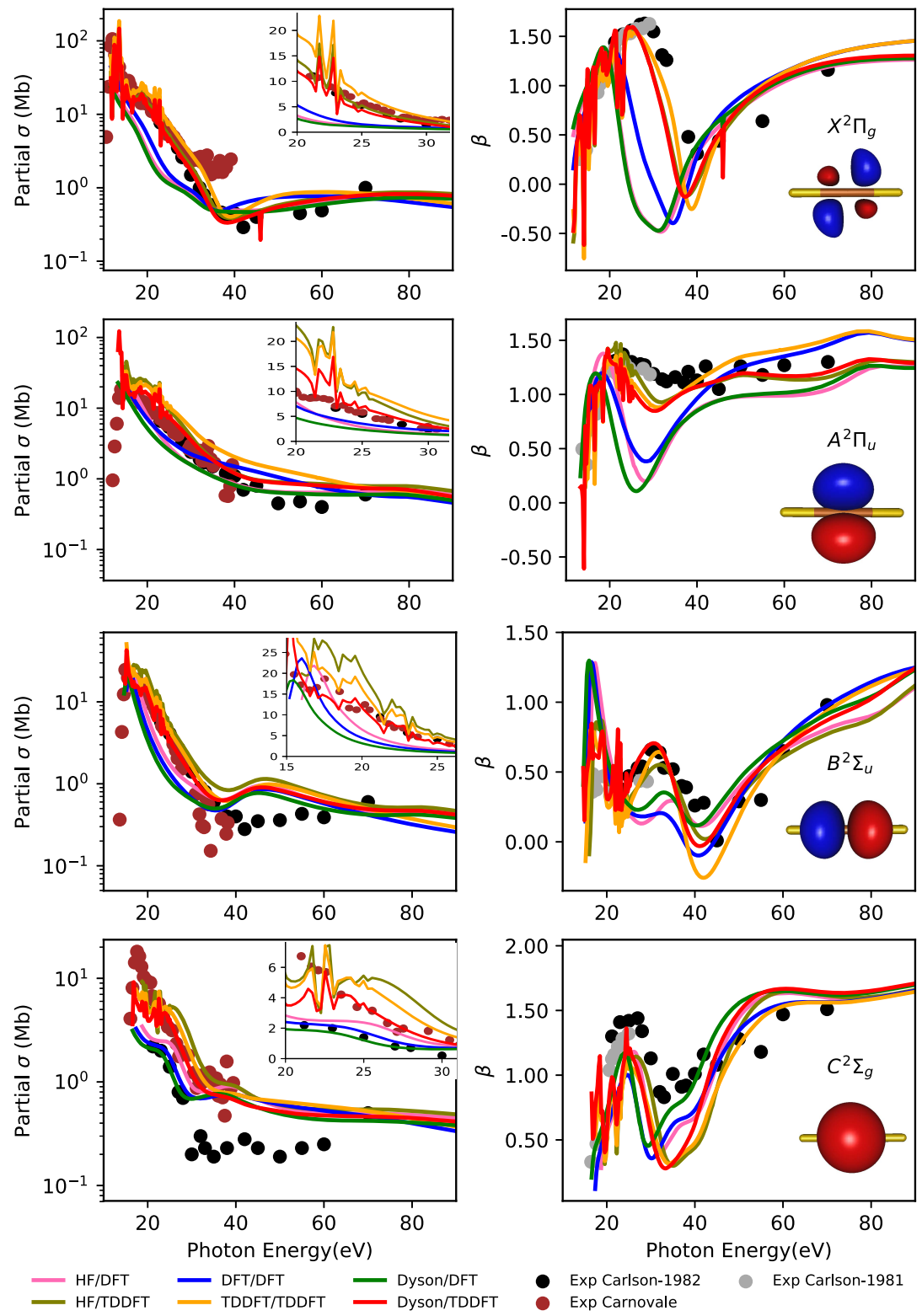

Figure 2. $\mathrm{CS}_{2}$ : partial cross section and the asymmetry parameter for the four lowest energy ionizations. Experimental results are taken from refs 69-71. Zoomed-in partial cross sections and the right EOM-CCSD Dyson orbitals are shown as insets.

4.2. Carbon Disulfide. For $\mathrm{CS}_{2}$, we studied the five lowest energy ionizations. The four outer-valence bands are quite straightforward, in the sense that they are solely characterized by ionization from a particular molecular orbital, with $R_{F}$ greater than 0.8 . The partial cross sections and asymmetry parameters are plotted as functions of photon energy in Figure 2. Table 3 summarizes our results for the ionization energies (and the EOM-CCSD pole strengths) versus the experiment. We refer to Figures $\mathrm{S} 1$ and $\mathrm{S} 3$ for renderings of the photoelectron spectrum.

In previous work, ${ }^{69}$ it was suggested that the strong enhancement of the cross section close to the threshold is due to the presence of a shape resonance. This suggestion is supported by the present approach, as a strong enhancement appears both in the DFT and TDDFT results. Curiously, however, the effect of TDDFT is significantly stronger in the highest occupied molecular orbital (HOMO) channel, compared to the following valence ionizations. This indicates that the dipole transition moments to the continuum from
Table 3. $\mathrm{CS}_{2}$ : Hartree-Fock Ionization Energies According to Koopmans' Theorem, LB94 Ground-State KS Eigenvalues, EOM-CCSD and CCSDR(3) Ionization Energies Versus the Experiment $^{a}$

$\begin{array}{lccccc}\text { state } & \text { HF } & \text { LCAO KS } & \text { EOM-CCSD } & R_{F} & \text { exp. } \\ X^{2} \Pi_{g} & 10.20 & 11.38 & 9.91(9.85) & 0.897 & 10.1 \\ A^{2} \Pi_{u} & 14.37 & 14.27 & 13.20(12.87) & 0.825 & 12.9 \\ B^{2} \Sigma_{u} & 15.78 & 15.07 & 14.45(14.25) & 0.873 & 14.6 \\ C^{2} \Sigma_{g} & 18.50 & 17.15 & 16.42(16.07) & 0.814 & 16.2 \\ D^{2} \Pi_{u} & & & 17.78(14.60) & 0.014 & 17.2 \\ { }^{2} \Sigma_{u} & 27.95 & 23.41 & 22.78(21.46) & 0.730 & \\ { }^{2} \Pi_{u} & & & 21.67(17.29) & 0.058 & \end{array}$

${ }^{a}$ The perturbatively corrected ionization energies, obtained using the CCSDR(3) method, are shown in parentheses. All energy values are in $\mathrm{eV}$. The EOM-CCSD pole strengths are reported in the second last column. The experimental results are taken from ref 69 . 

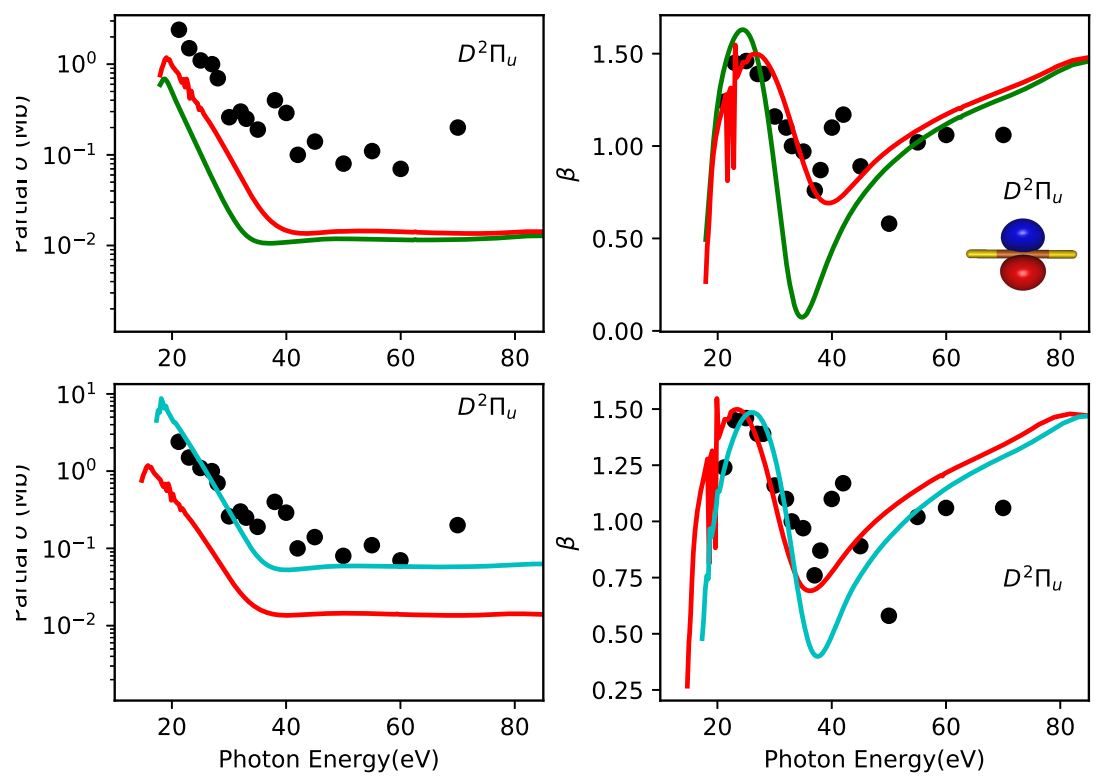

$2 /^{2} \Pi_{u}$ Dyson/DFT $2 /^{2} \Pi_{u}$ Dyson/TDDFT $-3 / 2 \Pi_{u}$ Dyson/TDDFT

Exp Carlson

Figure 3. $\mathrm{CS}_{2}$ : partial cross section and the asymmetry parameter of satellite states using EOM-CCSD Dyson orbitals. EOM-CCSD and CCSDR(3) ionization energies have been used in the top and bottom panels, respectively. Experimental results are taken from ref 69 . The right Dyson orbital is shown as an inset.

different initial orbitals are differently affected by interchannel coupling. Starting from the lowest energy ionization, i.e., from the HOMO $\left(2 \pi_{g}\right)^{-1}$, there is a remarkable difference between the partial cross sections computed using DFT and TDDFT continua in the near-threshold region (below $40 \mathrm{eV}$ ). The DFT spectrum underestimates the intensity in the region between 15 and $35 \mathrm{eV}$, with respect to the experiment. The TDDFT continuum, on the other hand, gives a substantial improvement. Moreover, several sharp structures are obtained close to the threshold due to autoionization resonances. These are generally washed out by vibrational motion in the experiment. Experimentally, these sharp peaks in the $X$ band, near-threshold, have been attributed to auto-ionizing Rydberg levels converging to higher-lying ionic states in addition to direct single-orbital ionizations. ${ }^{71}$ Then, we see that the TDDFT description of the continuum incorporates a new phenomenology, which is lacking in the DFT approach. However, both approaches are capable of capturing the Cooper minima. In the case of TDDFT, the agreement with the experiment is excellent. The asymmetry parameter for the $X$ band shows the typical nature as seen for atomic $S 3$ p orbital.

Next, for the $A$ band, all combinations of methods perform equally well in terms of matching with the experimental cross section. Astonishingly, all of the methods fail to capture the intensity increase in the near-threshold region. However, the accuracy of the various method differs markedly when looking at the asymmetry parameter. The Dyson/TDDFT approach reproduces the tail region of the spectrum above $50 \mathrm{eV}$ the best. The DFT continuum, on the other hand, overestimates the dip at the Cooper minima by about 1.0 units.

The third band arises dominantly due to electron ejection from the $5 \sigma_{u}$ orbital. The DFT continuum approach gives a rapidly decreasing cross-section profile. Even though the TDDFT continuum shows minimal deviation from the experimental profile up to $40 \mathrm{eV}$, it still lacks the narrow threshold feature. The importance of correlation in the boundstate description using Dyson orbitals is again demonstrated in the asymmetry parameter profile. The Dyson/TDDFT approach gives the closest representation of the experiment. The pure TDDFT computation overestimates the broad dip in the minima at $40 \mathrm{eV}$, whereas DFT continuum computations diminish the extent of increase at the maxima. Last, it is difficult to comment on the performance of the combination of methods in comparison to the experiment for band $C$, since the two experiments do not match with one another.

The outermost $\left(2 \pi_{g}\right)$ orbital is a pure combination of sulfur $3 p$ orbitals in a minimal basis set description, the following $2 \pi_{u}$ comprises some C $2 \mathrm{p}$, and even the next $\sigma_{u}$ is basically built from the same atomic orbitals, still dominated by sulfur $3 \mathrm{p}$. Still, the changes are clearly reflected in the cross section and more strongly in the $\beta$ parameter, and the variations are well captured by the theoretical approach.

According to the single-particle ionization pathway available from considering Koopmans' theorem, the fifth band should be due to ionization from the $4 \sigma_{u}$ orbital. However, the ionization energy for $\left(4 \sigma_{u}\right)^{-1}$ lies much higher in energy than the experimental band (27.95 eV in Table 3). At the EOM-CCSD level, the corresponding ${ }^{2} \Sigma_{u}$ state, with a significant amount of primary ionization, is found at $22.78 \mathrm{eV}$. In ref 69 , it was suggested that the fifth band is due to the main satellite peak of $\pi_{u}$ character. Using EOM-CCSD, we find the fifth band of $\Pi_{u}$ character at $17.78 \mathrm{eV}$, reasonably close to the experimental value of $17.2 \mathrm{eV}$. However, this ionization has a very low Dyson pole strength (0.014) and is predominantly of double-excitation character. An additional $\Pi_{u}$ ionization with a sizeable amount of primary single-excitation character is found at $21.67 \mathrm{eV}$, with a pole strength of 0.058 . Since states of double-excitation character are not so well described by EOM-CCSD (their energy is in general overestimated), we tried and applied a perturbative correction to the ionization energies using the CCSDR(3) method..$^{72}$ The results are also reported in Table 3.

In the two top panels of Figure 3, we present the partial cross section and asymmetry parameter for the first of the two EOM$\operatorname{CCSD} \Pi_{u}$ satellites and the two different continuum 


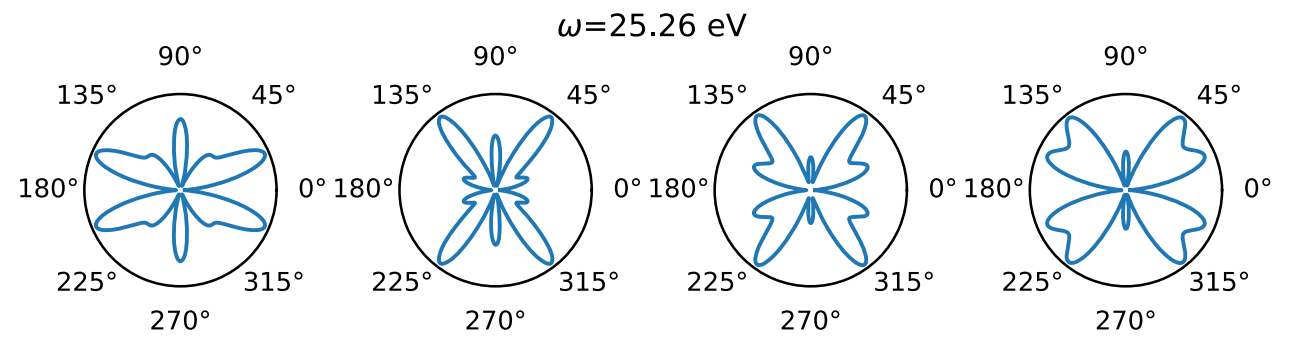

$\omega=36.42 \mathrm{eV}$
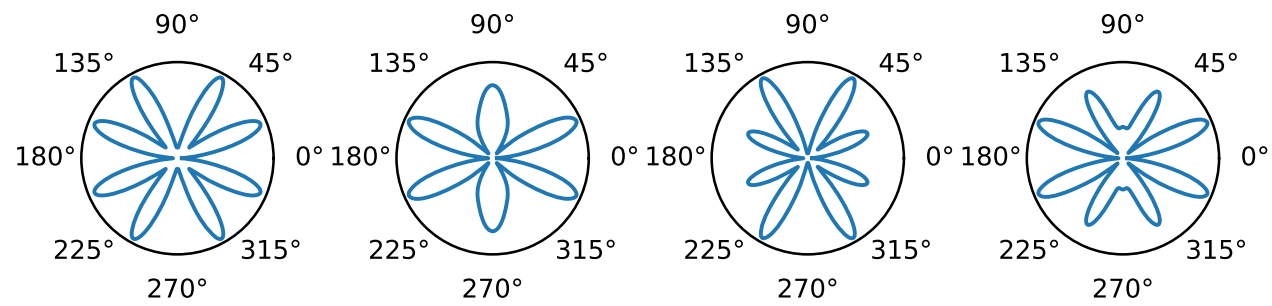

$\omega=50.02 \mathrm{eV}$
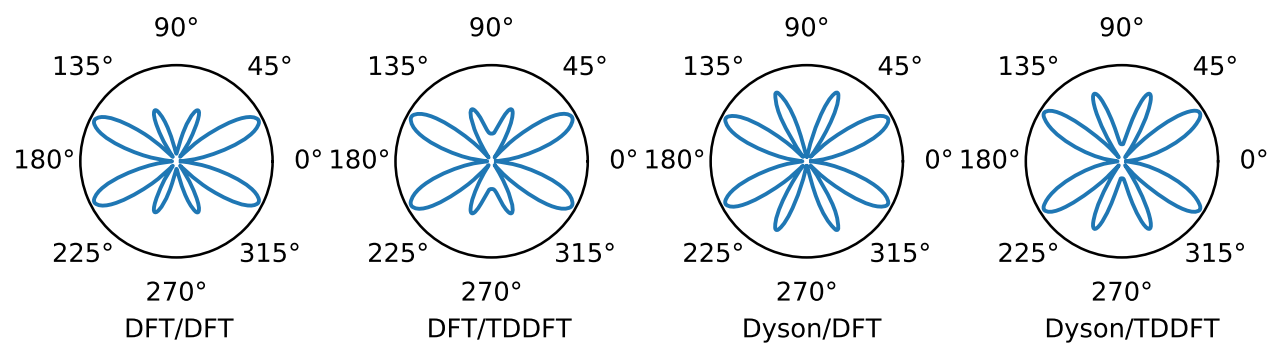

Figure 4. $\mathrm{CS}_{2}$ : MFPADs of the $X^{2} \Pi_{g}$ ionized state. The polarization vector is parallel to the molecular axis. All plots have been normalized to a common value for the maximum.

descriptions. The two panels at the bottom report the same data using the CCSDR(3) ionization energies and CCSD Dyson pole strengths for both $\Pi_{u}$ satellites and the TDDFT continuum. We refer to Figure S4 for the corresponding plots using the second $\Sigma_{u}$ ion.

If we describe the $D$ band using the data for the first EOM$\operatorname{CCSD} \Pi_{u}$ satellite, the partial cross section is underestimated by one order of magnitude owing to the significantly small $R_{F}$ value. This is considerably improved when we use the EOM Dyson orbital of the second $\Pi_{u}$ satellite. The asymmetry parameter is quite accurately reproduced using the Dyson orbitals of the $\Pi_{u}$ symmetry satellites, contrary to what is obtained with the higherenergy $\Sigma_{u}$ one. This corroborates the analysis in the experimental study. ${ }^{69}$ It is also quite evident from Figure 3 that the TDDFT continuum orbitals better represent the actual scenario than the DFT continuum orbitals.

Finally, as a signature of the final states, we have computed the molecular frame angular distributions, as shown in Figures 4 and S5-S10. Our choice of photon energies $\omega$ was driven by the desire to investigate the change in the angular distribution near the Cooper minimum at around $37 \mathrm{eV}$. In the case of the parallel orientation for the $X$ band, the emission profiles obtained from the DFT and the TDDFT treatments of the continuum are quite different, although maintaining a butterfly-like shape. Due to the symmetry of the molecule, the forward and backward emissions are mirror images of one another.

4.3. Nickel Bisallyl Complex. We now investigate the giant correlation effects necessary for the description of transition metal compounds. The $\mathrm{Ni}\left(\mathrm{C}_{3} \mathrm{H}_{5}\right)_{2}$ complex, see Figure 5, is an interesting molecule due to the strong disagreement between

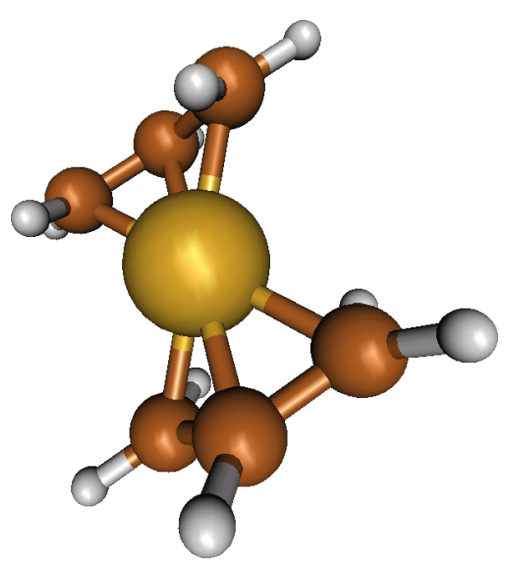

Figure 5. Nickel-bisallyl complex.

experimental evidence and results from Hartree-Fock molecular orbital calculations. The molecule is a classic example of the complete breakdown of Koopmans' theorem. It exhibits large relaxation effects, especially in the case of ionization from the $\mathrm{Ni}$ $\mathrm{d}$ orbitals. The $\mathrm{d}$ orbitals are embedded deep within the valence shell at the Hartree-Fock level, but become the lowest bound at the $\triangle$ SCF level, which provides relaxation energies up to $10 \mathrm{eV}$ for such ionizations. ${ }^{73}$ This is partly exaggerated because of the lack of correlation, which alleviates the strong interelectronic repulsion of the compact $d$ shell. Despite the intense effort in the past, the assignment of the spectrum is still not firmly settled. It is generally accepted that the first three bands comprise both four metal $3 \mathrm{~d}\left({ }^{2} A_{g}\right.$ and $\left.{ }^{2} B_{g}\right)$ ionizations, and the outermost $\pi$ allyl 
ionization $\left(7 a_{u}\right)$, but the specific ordering is debated. A careful experimental study, based on trends among substituted allyl compounds, ${ }^{74}$ reinforced by later synchrotron radiation experiments, ${ }^{75,76}$ favors metal d orbital ionization as the outermost, while the most elaborate theoretical calculations give allyl $7 a_{u}$ as the least bound, but again details vary considerably.

In Table 4, we report a comparison between the experiment and computed ionization energies obtained from Koopmans'

Table 4. $\mathrm{Ni}\left(\mathrm{C}_{3} \mathrm{H}_{5}\right)_{2}$ : Hartree-Fock Ionization Energies According to Koopmans' Theorem, EOM-CCSD Ionization Energies and Pole Strengths, and Experimental Energies of the Peak Maxima ${ }^{a}$

\begin{tabular}{|c|c|c|c|c|c|c|}
\hline \multirow[b]{2}{*}{ band } & \multirow[b]{2}{*}{ state } & \multirow[b]{2}{*}{ MO } & \multirow{2}{*}{$\begin{array}{c}\text { K.T. } \\
\text { I.E. }\end{array}$} & \multicolumn{2}{|c|}{ EOM-CCSD } & \multirow[b]{2}{*}{ exp..$^{74}$} \\
\hline & & & & I.E. & $R_{F}$ & \\
\hline \multirow[t]{2}{*}{1} & ${ }^{2} A_{u}$ & $7 a_{u}$ & 7.73 & 7.20 & 0.898 & $7.64 / 7.79$ \\
\hline & ${ }^{2} A_{g}$ & $13 a_{g}$ & 11.75 & 7.22 & 0.875 & \\
\hline \multirow[t]{2}{*}{2} & ${ }^{2} B_{g}$ & $6 b_{g}$ & 8.38 & 7.44 & 0.865 & $7.95 / 8.15$ \\
\hline & ${ }^{2} A_{g}$ & $12 a_{g}$ & 14.01 & 7.86 & 0.870 & \\
\hline 3 & ${ }^{2} A_{g}$ & $11 a_{g}$ & 14.12 & 8.46 & 0.868 & 8.52 \\
\hline 4 & ${ }^{2} B_{g}$ & $5 b_{g}$ & 14.09 & 8.81 & 0.842 & 9.38 \\
\hline 5 & ${ }^{2} B_{u}$ & $11 b_{u}$ & 11.81 & 10.80 & 0.879 & 10.36 \\
\hline 6 & ${ }^{2} A_{g}$ & $10 a_{g}$ & 15.65 & 12.18 & 0.857 & 11.48 \\
\hline \multirow[t]{4}{*}{7} & ${ }^{2} A_{u}$ & $6 a_{u}$ & 14.53 & 13.12 & 0.891 & 12.6 \\
\hline & ${ }^{2} B_{u}$ & $10 b_{u}$ & 14.67 & 13.27 & 0.892 & \\
\hline & ${ }^{2} B_{g}$ & $4 b_{g}$ & 14.83 & 13.35 & 0.877 & \\
\hline & ${ }^{2} A_{g}$ & $9 a_{g}$ & 16.44 & 13.45 & 0.892 & \\
\hline \multirow[t]{2}{*}{8} & ${ }^{2} A_{u}$ & $5 a_{u}$ & 16.35 & 14.68 & 0.876 & 14.2 \\
\hline & ${ }^{2} B_{g}$ & $3 b_{g}$ & 17.31 & 14.91 & 0.874 & \\
\hline \multirow[t]{8}{*}{9} & ${ }^{2} B_{u}$ & & & 16.01 & 0.263 & 15.6 \\
\hline & ${ }^{2} B_{u}$ & $9 b_{u}$ & 17.85 & 16.08 & 0.602 & \\
\hline & ${ }^{2} A_{u}$ & & & 16.39 & 0.004 & \\
\hline & ${ }^{2} A_{g}$ & $8 a_{g}$ & 19.05 & 16.41 & 0.861 & \\
\hline & ${ }^{2} B_{u}$ & & & 16.48 & 0.007 & \\
\hline & ${ }^{2} B_{u}$ & & & 16.98 & 0.006 & \\
\hline & ${ }^{2} B_{g}$ & $2 b_{g}$ & 24.98 & 17.29 & 0.018 & \\
\hline & ${ }^{2} A_{u}$ & & & 17.67 & 0.002 & \\
\hline 10 & ${ }^{2} A_{g}$ & $7 a_{g}$ & 21.47 & 18.23 & 0.034 & 17.9 \\
\hline
\end{tabular}

${ }^{a}$ All energies are in eV. Experimental results are taken from ref 74 .

theorem at the Hartree-Fock level and from EOM-IP-CCSD/ cc-pV $(\mathrm{D}+\mathrm{d}) \mathrm{Z}(\mathrm{Ni}) / \mathrm{DZP}(\mathrm{C}, \mathrm{H})$ calculations. This choice of basis set is a compromise between accuracy and computational cost. We note, however, that using the aug-cc-pVDZ basis set on all atoms, the photoelectron spectrum does not vary significantly; see Figure S13 for more details. We shall not discuss the assignment in detail; however, note that while we agree in indicating $7 a_{u}$ as the outermost ionization, it is almost degenerate with the following metal $3 \mathrm{~d}$ bands, and this is probably the reason for the difficulty to ascertain the correct ordering. Looking at the calculated spectrum (Figure 6), we observe that although the outermost structure is not perfectwith bands 2 and 3 that should be closer to band 1 and too large a gap between bands 4 and 5-it is overall quite reasonable. We also obtain a tiny hump region between bands 9 and 10, in the range of photon energies from 16.9 to $17.8 \mathrm{eV}$, arising due to transitions having very low Dyson square norm. These targetbound ionization states are also an outcome of correlation included in the EOM-CCSD Dyson orbital framework.

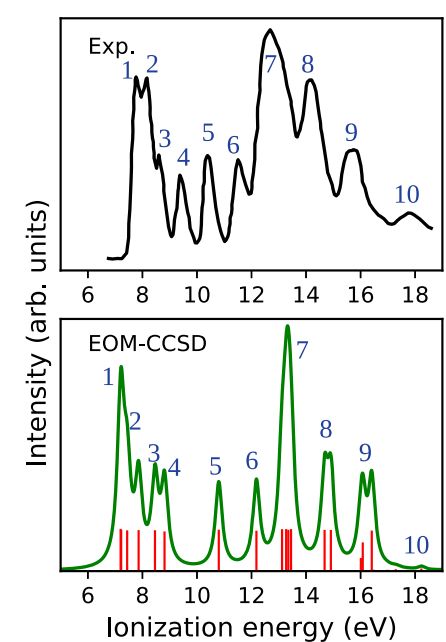

Figure 6. $\mathrm{Ni}\left(\mathrm{C}_{3} \mathrm{H}_{5}\right)_{2}$ : photoelectron spectrum. The experimental spectrum was redigitized from ref 74 .

The lowest IEs are underestimated, and then, progressively overestimated at the high-energy side, probably partly due to the relatively modest basis set employed and incomplete recovery of correlation. Still, differential correlation contributions, of about 5-6 eV for the outer ${ }^{2} A_{g}$ and ${ }^{2} B_{g}$ states, compared to less than 1 $\mathrm{eV}$ for the ${ }^{2} A_{u}$ and ${ }^{2} B_{u}$ ones, are completely recovered. It is also interesting to note the strong mixing of HF MOs in the Dyson orbitals. Even if square norms of the Dyson orbitals are still above 0.8 , suggesting the validity of the one-electron picture, a large orbital mixing is observed. While the $7 a_{u}^{-1}$ Dyson orbital is practically equal to the $7 a_{u} \mathrm{MO}$ (apart from the reduced norm), the (renormalized) composition of the left $13 a_{g}^{-1}$ Dyson is $-0.797 \cdot 13 a_{g}+0.410 \cdot 9 a_{g}+0.300 \cdot 10 a_{g}+0.273 \cdot 12 a_{g}$ MOs; the $12 a_{g}^{-1}$ Dyson is $0.724 \cdot 11 a_{g}-0.598 \cdot 12 a_{g}+0.205 \cdot 10 a_{g}+0.155$. $9 a_{g}+0.136 \cdot 8 a_{g}$, and so on.

While this "hole mixing" or "orbital rotation" upon ionization has been discussed long ago, ${ }^{77}$ it has remained a purely theoretical construct, in the absence of experimental verification. Only transition properties that involve the Dyson orbital can actually probe such an effect, and a prominent one is through photoionization observables. Some representative cross section and asymmetry parameter results for selected ionizations are reported in Figures 7 and 8. A full set is available in Figures S14S33.

The analysis of our present results, together with previous ones, highlights four important points:

1. Comparison of results relative to HF, DFT, and Dyson orbitals, all with the same DFT continuum, reported in Figure 7 , illustrates the importance of correlation in the bound states. The effect is most dramatic in the $\beta$ parameter. In the case of ionization of the nominal $13 a_{g}$ orbital, HF and Dyson results are very different, illustrating the large reorganization upon ionization. The effect is less marked, but still important, on the cross section. The diminished sensitivity of the cross section is probably due to the fact that it is dominated by the metal $3 \mathrm{~d}$ component, which remains major in both descriptions. Similar results are obtained also for most (but not all) $a_{g}$ ionizations (see Figures S14-S20). Analogous considerations hold for the $b_{g}$ ionizations, see, e.g., $6 b_{g}$ and $5 b_{g}$, which also suggest that upon ionization they mostly exchange role, as HF results for $6 b_{g}$ resemble 

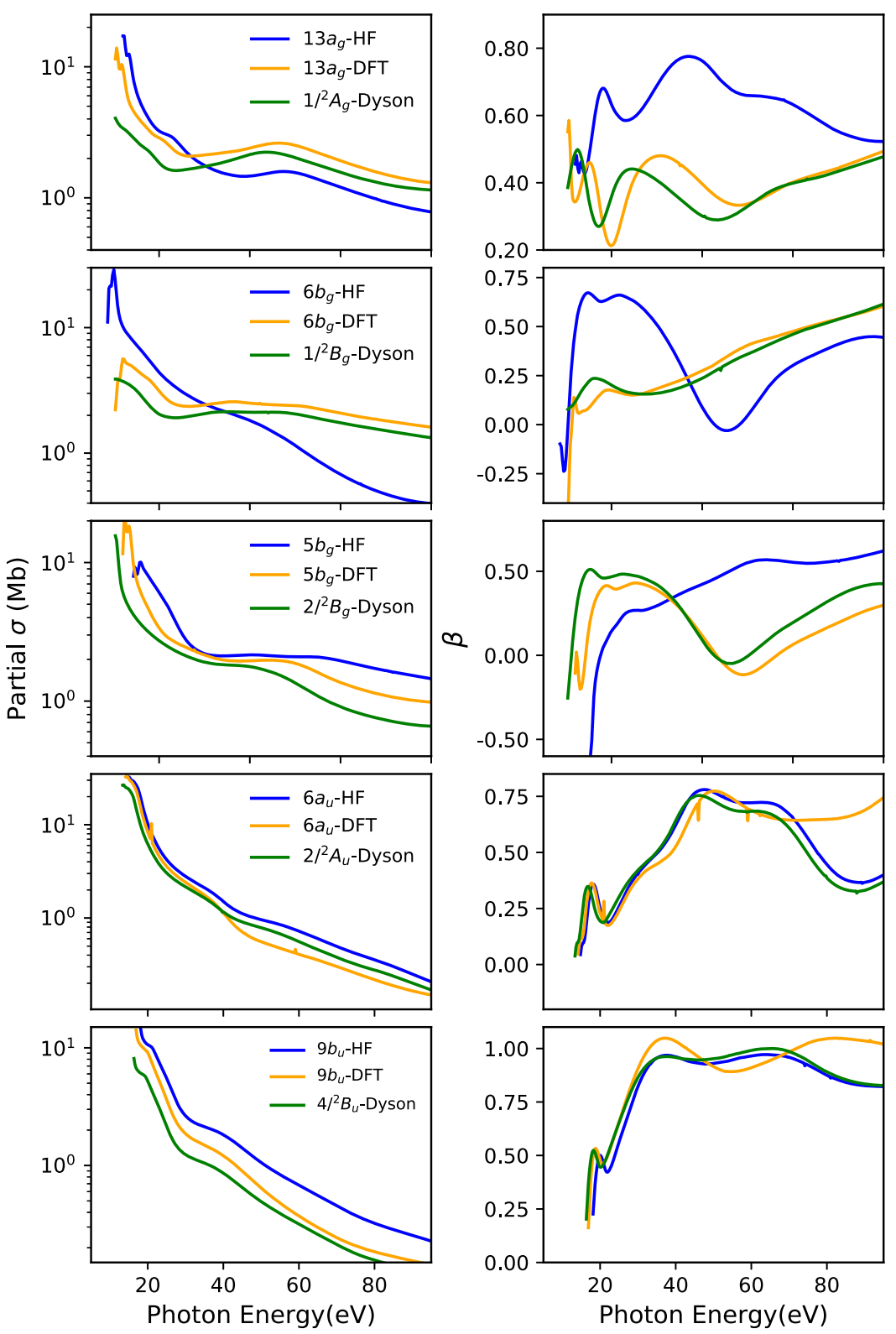

Figure 7. $\mathrm{Ni}\left(\mathrm{C}_{3} \mathrm{H}_{5}\right)_{2}$ : partial cross sections and asymmetry parameters for particular bound-state ionizations. The B-spline DFT has been used for the description of the continuum orbital.

those of the $2 /{ }^{2} B_{g}$ Dyson (and $5 b_{g}$ DFT), and vice versa. This is again validated by the fact that the major molecular orbital contributing to $1 /{ }^{2} B_{g}$ and $2 /{ }^{2} B_{g}$ Dyson orbitals are indeed $5 b_{g}$ and $6 b_{g}$, respectively. On the other hand, for MOs essentially localized on the organic moiety, the HF and Dyson results are close. We have here chosen $6 a_{u}$ and $9 b_{u}$ for illustration, but the same holds for all $a_{u}$ and $b_{u}$ ionizations, that have no metal $3 \mathrm{~d}$ component, as well as for some inner $a_{g}$ and $b_{g}$ ionizations (see Figures S25S33).

2 Still with reference to Figure 7, it is interesting to compare also the results relative to initial DFT orbitals. As can be seen for $13 a_{g}, 6 b_{g}$, and $5 b_{g}$, in this case, the results for DFT orbitals are much closer to the Dyson orbitals' ones. It is a clear indication of the superior performance of DFT compared to HF for the description of $\mathrm{d}$ orbitals in transition metal complexes, a well-known fact. It appears that the exchange-correlation potential in DFT gives a more balanced description of the interelectronic interaction, while a differential correlation between compact $\mathrm{d}$ versus $\mathrm{s}, \mathrm{p}$ orbitals is much larger in $a b$ initio. Curiously, however, DFT performs more poorly for some ligand orbitals, as is clearly seen (especially at higher energies, see Figures S26 and S32) for $6 a_{u}$ and $9 b_{w}$, where HF is closer to Dyson. This behavior offers interesting insights into the nature of DFT orbitals and may suggest specific areas of improvement, complementary to other global properties.

3. The large effect of interchannel coupling has been already highlighted in $\mathrm{H}_{2} \mathrm{~S}$ and $\mathrm{CS}_{2}$. Again, this is very specific. In the case of nickel bisallyl, it turns out to be sizable only close to the threshold, and, depending on the orbital, of modest or minor importance, as can be seen for the same orbitals in Figure 8. But the striking effect is the appearance of autoionization resonances in the region of metal $3 \mathrm{p} \rightarrow 3 \mathrm{~d}$ excitation, at about $70 \mathrm{eV}$. This is a very 

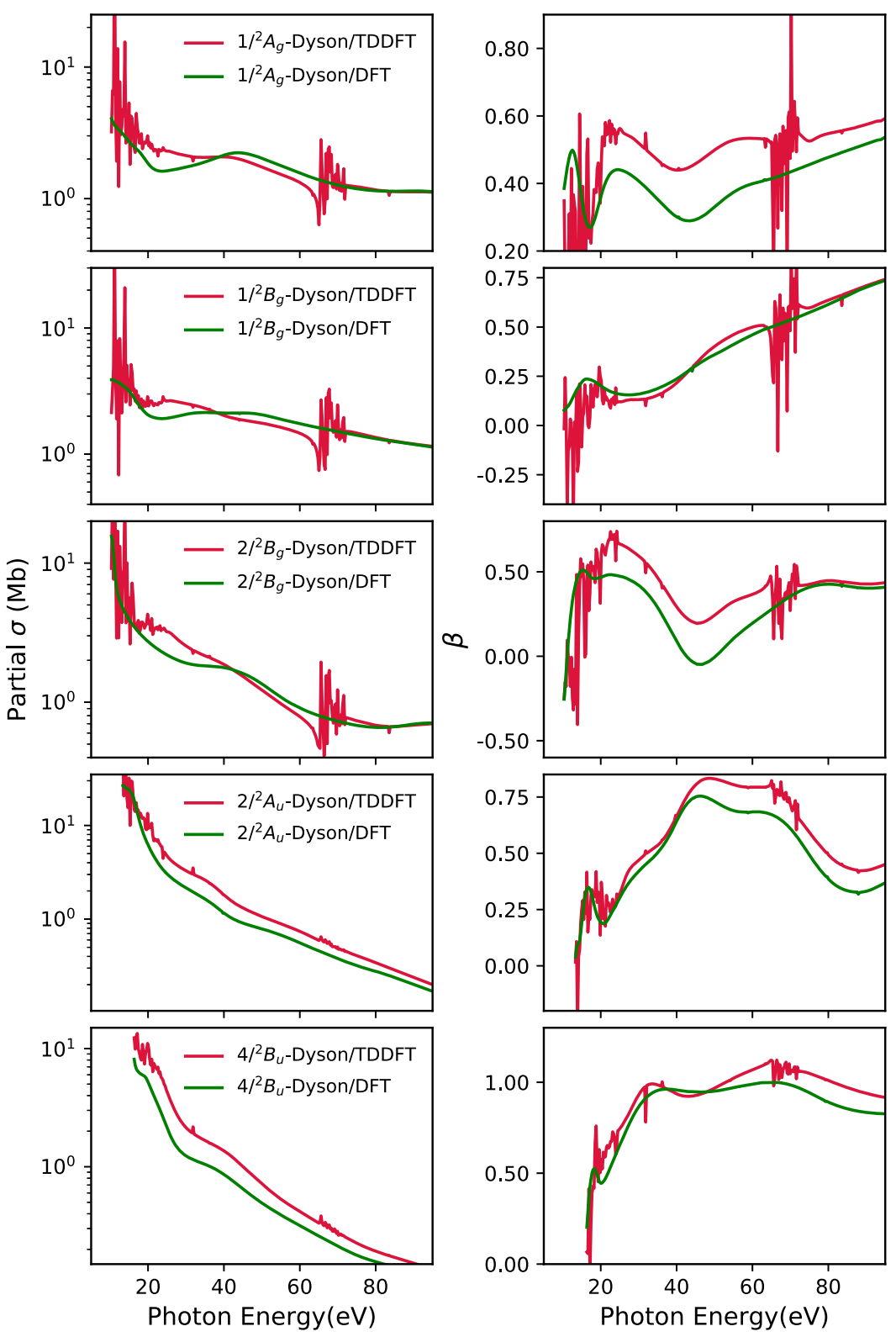

Figure 8. $\mathrm{Ni}\left(\mathrm{C}_{3} \mathrm{H}_{5}\right)_{2}$ : partial cross section and asymmetry parameters for particular bound-state ionizations. EOM-CCSD Dyson orbitals have been used for the description of the bound part.

strong signature of the metal $d$ participation in the orbital ionized, and, in fact, it is strongly present in the $a_{g}$ and $b_{g}$ ionizations relative to $\mathrm{Ni} 3 \mathrm{~d}$ orbitals, and are instead barely visible in the $a_{u}$ and $b_{u}$ ligand bands, which can have no metal $3 \mathrm{~d}$ content, and appear only via weak continuum-continuum coupling with the other channels.

4. Clearly, photoionization observables are a powerful tool for spectral assignment. In fact, this has been used since the inception of photoelectron spectroscopy as a change in band intensities in going from $\mathrm{HeI}$ to HeII radiation, and to X-rays, or as differences in the $\beta$ behavior between $\sigma$ and $\pi$ orbitals, but it has become much more informative with the advent of tunable synchrotron radiation. It is well known that upon comparing ionization cross-section profiles from $2 p$ orbitals of the second-row elements with $3 \mathrm{~d}$ orbitals of transition metals, the former displays a rapidly decaying cross section with a higher value near the threshold. Based on this, we can comment from Figure 9 that the outermost bands are due to ionizations from the $3 \mathrm{~d}$ orbitals of the $\mathrm{Ni}$ atom, except that corresponding to the $7 a_{u}$ orbital. This Dyson orbital is similar to the $\pi$ orbital on the $\mathrm{C}_{3} \mathrm{H}_{5}$ moiety. On the other hand, all of the ionization pathways constituting bands 5-10 mimic the profile for the $2 \mathrm{p}$ orbital of the $\mathrm{C}$ atom. Thus, these are of ligand origin.

As a single example, we report, in Figure 10, the ratio of the $7 a_{u}$ to $13 a_{g}$ cross sections, which are expected to be the two outermost ionizations. A careful high-resolution experiment could probably assess which is the HOMO band if they are not practically degenerate, and in general to disentangle the assignment of the ionizations composed of in bands 1-3. A more detailed analysis based on pure DFT/TDDFT results was presented in a previous study. ${ }^{78}$ 


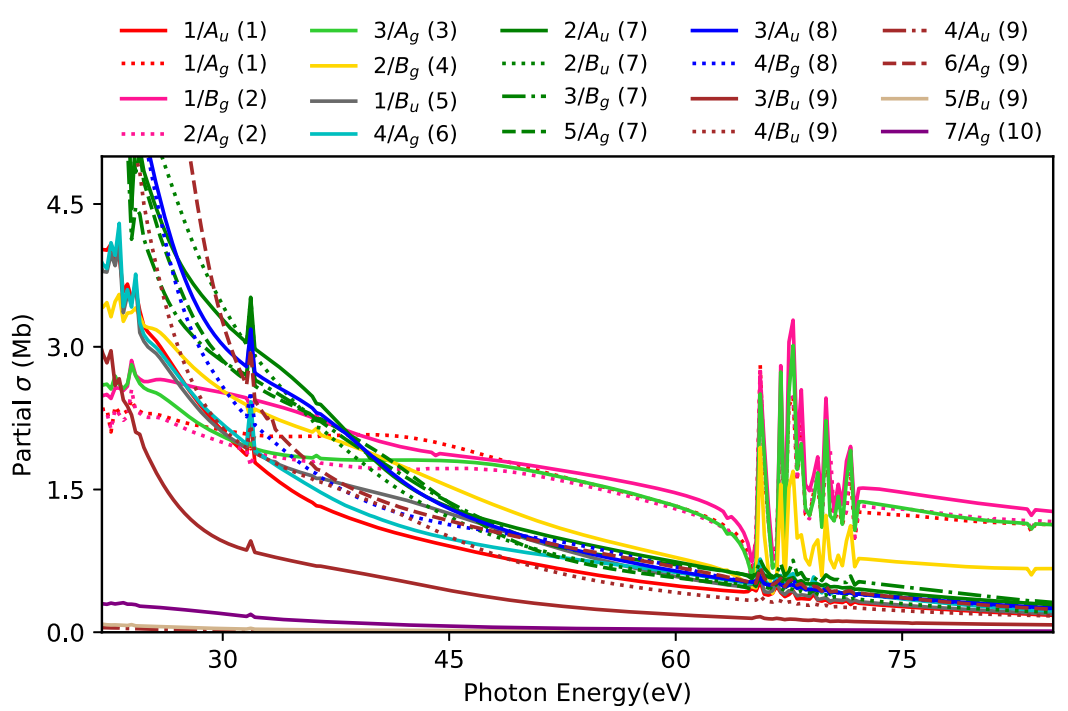

Figure 9. $\mathrm{Ni}\left(\mathrm{C}_{3} \mathrm{H}_{5}\right)_{2}$ : partial cross section of the ionizations for bands 1-10, computed using the EOM-CCSD Dyson/TDDFT approach. The band to which the ionization belongs is given in parentheses.

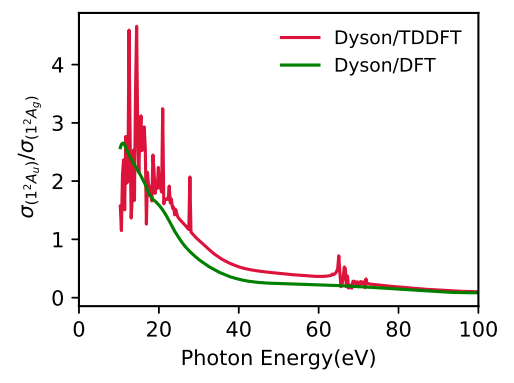

Figure 10. $\mathrm{Ni}\left(\mathrm{C}_{3} \mathrm{H}_{5}\right)_{2}$ : ratio of a partial cross section of the ionizations from the two outermost bound channels.

\section{CONCLUSIONS}

In summary, our devised methodology, coupling EOM-CCSD Dyson orbital coefficients to describe the bound-state character of the target system and B-spline TDDFT continuum orbitals, gives a precise depiction of the photoionization properties. The developed method acts as a more accurate extension of the EOM-CCSD Dyson/KS-DFT approach described earlier. ${ }^{16}$ However, this extra noniterative step over KS-DFT is generally necessary only for reproducing highly sophisticated experimental observations. We observe that for the case of ionizations from the outer-valence orbitals originating from $2 s, 2 p$ orbitals of second-row atoms, only a slight improvement is seen upon using TDDFT continuum over DFT continuum orbitals. Although, as all of the corresponding Dyson orbitals are practically equivalent to the molecular orbital considered for Hartree-Fock or DFT calculation, no drastic improvement is noticed. However, the formalism is an absolute necessity in the case of treatment of interchannel couplings, the precise positioning of Cooper minima, and to account for correlated ionization states, like satellite states, which are absent in Koopmans' treatment. Moreover, our approach accounts for auto-ionization resonances lacking in its DFT equivalent. We also manifest that in the case of transition metal complexes, Koopmans' theorem often fails due to large correlation and relaxation effects, which also cause dramatic changes of Dyson orbitals with respect to $a b$ initio ones. Interestingly, in such cases, DFT orbitals are much closer to Dyson than to HF ones. The opposite is found, however, for other ionizations. The interpretation of photo- electron data is then possible only upon using a highly correlated bound target state description, as exemplified in the case of nickel bisallyl complex.

The approach presented here is completely general and computationally efficient. It allows for the calculation of all photoionization dynamical variables. We here present results for partial cross sections, branching ratios, asymmetry parameters, and molecular frame photoelectron angular distributions. It is seen that the asymmetry parameter is the one most significantly improved by our current approach. Based on the molecules studied, we also conclude that generally molecules with third (or higher)-period elements, are better described by the EOMCCSD Dyson/TDDFT approach. The appearance of the Cooper minima in the asymmetry parameters is very characteristic of third-row atoms (here, S 3p orbital).

Furthermore, theoretical advancements are possible building on this formalism, like the inclusion of higher-order correlation effects in the treatment of the bound states. Open-shell systems can, in principle, also be treated within the proposed formalism and will be the subject of future studies. The method is also suitable for extension to encompass nuclear dynamic problems to describe the vibrational resolution of the experimental spectra. Another interesting application can be for the case of time-resolved experiments, as the photoionization process is highly sensitive to the bound-state nature of the ionizing orbital.

Finally, although we have not considered satellite states in detail in this work, photoionization observables are a powerful signature of such states, giving information on the nature of their electronic configuration and parentage of primary states, if any. Since they are strictly forbidden at the one-particle level, they are a direct probe of correlation effects and a challenging task for highly correlated theoretical approaches. In fact, while originally raising great interest, the inadequacy of the theoretical tools of the time and experimental difficulty in accurately measuring such often weak and poorly resolved features, caused decline in interest. Present facilities are now at a point that a renewed attack on the problem, both experimentally and theoretically, is warranted. Since the EOM-CCSD approach employed here is still only partly adequate, we have considered only one wellcharacterized satellite in $\mathrm{CS}_{2}$, where already a rather good reproduction of the experimental data is afforded. More detailed 
studies, including branching ratios with primary states, of the several satellites that are detected in all these molecules, may give a more precise characterization of the nature of the multielectron excitations and the improvement of the theoretical tools needed for a quantitative description. Among these improvements is a more accurate account of double-excitation effects, as our preliminary results using a perturbatively corrected ionization energy of the satellite state of $\mathrm{CS}_{2}$ indicate. Work in this direction is in progress.

\section{ASSOCIATED CONTENT}

\section{SI Supporting Information}

The Supporting Information is available free of charge at https://pubs.acs.org/doi/10.1021/acs.jctc.1c00303.

Computed photoelectron spectra versus experiment; comparison of $R_{F}$ computed using different definitions; characterizing the satellite band of $\mathrm{CS}_{2}$; MFPADs of $\mathrm{CS}_{2}$; results and discussion for $\mathrm{C}_{3} \mathrm{O}_{2}$ and $\mathrm{C}_{3} \mathrm{~S}_{2}$; for $\mathrm{Ni}\left(\mathrm{C}_{3} \mathrm{H}_{5}\right)_{2}$ : EOM-CCSD/aug-cc-pVDZ PES and individual target bound-state partial cross section and asymmetry parameters; geometries (Cartesian coordinates) of all molecules studied (PDF)

\section{AUTHOR INFORMATION}

\section{Corresponding Authors}

Sonia Coriani - DTU Chemistry, Technical University of Denmark, DK-2800 Kgs. Lyngby, Denmark; NTNUNorwegian University of Science and Technology, N-7491 Trondheim, Norway; (1) orcid.org/0000-0002-4487-897X; Email: soco@kemi.dtu.dk

Piero Decleva - Istituto Officina dei Materiali IOM-CNR and Dipartimento di Scienze Chimiche e Farmaceutiche, Università degli Studi di Trieste, I-34121 Trieste, Italy; (1) orcid.org/ 0000-0002-7322-887X; Email: decleva@units.it

\section{Author}

Torsha Moitra - DTU Chemistry, Technical University of Denmark, DK-2800 Kgs. Lyngby, Denmark; 이이.org/ 0000-0001-7228-7678

Complete contact information is available at:

https://pubs.acs.org/10.1021/acs.jctc.1c00303

\section{Notes}

The authors declare no competing financial interest.

\section{ACKNOWLEDGMENTS}

S.C. thanks Dr. John Stanton (University of Florida) for useful discussion. The authors acknowledge support from the Marie Skłodowska-Curie European Training Network COmputational Spectroscopy In Natural Sciences and Engineering (COSINE), Grant Agreement no. 765739. S.C. acknowledges financial support from the Independent Research Fund Denmark DFFFNU RP2 (grant no. 7014-00258B) and from the Research Council of Norway through FRINATEK project 275506, Theolight. Finally, the COST Action CA18222, Attochem, is acknowledged.

\section{REFERENCES}

(1) Langhoff, P. Stieltjes imaging of atomic and molecular photoabsorption profiles. Chem. Phys. Lett. 1973, 22, 60-64.

(2) Langhoff, P. W.; Corcoran, C. T. Stieltjes imaging of photoabsorption and dispersion profiles. J. Chem. Phys. 1974, 61, 146-159.
(3) Langhoff, P. W.; Corcoran, C. T.; Sims, J. S.; Weinhold, F.; Glover, R. M. Moment-theory investigations of photoabsorption and dispersion profiles in atoms and ions. Phys. Rev. A 1976, 14, 1042-1056.

(4) Müller-Plathe, F.; Diercksen, G. H. Molecular Photoionisation Cross Sections by Moment Theory. An Introduction. In Electronic Structure of Atoms, Molecules and Solids. Proceeding of the II Escola Brasileira de Estructure Eletronica, Olinda, Brazil, July 17-22, 1989; Canuto, S.; D’Albuquerque e Castro, J.; Paixao, F. J., Eds.; World Scientific: Olinda, Brazil, 1990; pp 1-29.

(5) Cukras, J.; Coriani, S.; Decleva, P.; Christiansen, O.; Norman, P. Photoionization cross section by Stieltjes imaging applied to coupled cluster Lanczos pseudo-spectra. J. Chem. Phys. 2013, 139, No. 094103.

(6) Ruberti, M.; Yun, R.; Gokhberg, K.; Kopelke, S.; Cederbaum, L. S.; Tarantelli, F.; Averbukh, V. Total Molecular Photoionization CrossSections by Algebraic Diagrammatic Construction-Stieltjes-Lanczos method: Benchmark Calculations. J. Chem. Phys. 2013, 139, No. 144107.

(7) Tenorio, B. N. C.; Nascimento, M. A. C.; Coriani, S.; Rocha, A. B. Coupled Cluster Study of Photoionization and Photodetachment Cross Sections. J. Chem. Theory Comput. 2016, 12, 4440-4459.

(8) Tenorio, B. N. C.; Moitra, T.; Nascimento, M. A. C.; Rocha, A. B.; Coriani, S. Molecular inner-shell photoabsorption/photoionization cross sections at core-valence-separated coupled cluster level: Theory and examples. J. Chem. Phys. 2019, 150, No. 224104.

(9) Tenorio, B. N. C.; Chaer Nascimento, M. A.; Rocha, A. B.; Coriani, S. Lanczos-based equation-of-motion coupled-cluster singlesand-doubles approach to the total photoionization cross section of valence excited states. J. Chem. Phys. 2019, 151, No. 184106.

(10) Tenorio, B. N. C.; Coriani, S.; Rocha, A. B.; Nascimento, M. A. C. Molecular Photoionization and Photodetachment Cross Sections Based on $\mathrm{L}^{2}$ Basis Sets: Theory and Selected Examples. In Advances in Methods and Applications of Quantum Systems in Chemistry, Physics, and Biology; Glushkov, A. V., Khetselius, O. Y., Maruani, J., Brändas, E. Eds.; Springer International Publishing: Cham, 2021; pp 151-179.

(11) Gokhberg, K.; Vysotskiy, V.; Cederbaum, L. S.; Storchi, L.; Tarantelli, F.; Averbukh, V. Molecular photoionization cross sections by Stieltjes-Chebyshev moment theory applied to Lanczos pseudospectra. J. Chem. Phys. 2009, 130, No. 064104.

(12) Ruberti, M.; Averbukh, V.; Decleva, P. B-spline algebraic diagrammatic construction: Application to photoionization crosssections and high-order harmonic generation. J. Chem. Phys. 2014, 141, No. 164126.

(13) Oana, C. M.; Krylov, A. I. Cross sections and photoelectron angular distributions in photodetachment from negative ions using equation-of-motion coupled-cluster Dyson orbitals. J. Chem. Phys. 2009, 131, No. 124114.

(14) Gozem, S.; Gunina, A. O.; Ichino, T.; Osborn, D. L.; Stanton, J. F.; Krylov, A. I. Photoelectron Wave Function in Photoionization: Plane Wave or Coulomb Wave? J. Phys. Chem. Lett. 2015, 6, 45324540.

(15) Oana, C. M.; Krylov, A. I. Dyson orbitals for ionization from the ground and electronically excited states within equation-of-motion coupled-cluster formalism: Theory, implementation, and examples. J. Chem. Phys. 2007, 127, No. 234106.

(16) Moitra, T.; Ponzi, A.; Koch, H.; Coriani, S.; Decleva, P. Accurate Description of Photoionization Dynamical Parameters. J. Phys. Chem. Lett. 2020, 11, 5330-5337.

(17) Burke, P. G. R-matrix Theory of Atomic Collisions: Application to Atomic, Molecular and Optical Processes; Springer, 2011.

(18) Mašín, Z.; Benda, J.; Gorfinkiel, J. D.; Harvey, A. G.; Tennyson, J. UKRmol+: A suite for modelling electronic processes in molecules interacting with electrons, positrons and photons using the R-matrix method. Comput. Phys. Commun. 2020, 249, No. 107092.

(19) Amar, M. B.; Farnoux, F. C. Numerical solution of the LippmannSchwinger equations in photoemission: application to xenon. J. Phys. B: At. Mol. Phys. 1983, 16, 2339-2358.

(20) Lucchese, R. R.; Takatsuka, K.; McKoy, V. Applications of the Schwinger variational principle to electron-molecule collisions and molecular photoionization. Phys. Rep. 1986, 131, 147-221. 
(21) Rescigno, T. N.; McCurdy, C. W.; Orel, A. E.; Lengsfield, B. H. The Complex Kohn Variational Method. In Computational Methods for Electron-Molecule Collisions; Huo, W. M.; Gianturco, F. A., Eds.; Springer US: Boston, MA, 1995; pp 1-44.

(22) Brosolo, M.; Decleva, P.; Lisini, A. LCAO expansion in a spline basis for accurate variational determination of continuum wavefunctions. Applications to $\mathrm{H}_{2}^{+}$and $\mathrm{HeH}^{2+}$. Chem. Phys. 1994, 181, $85-95$.

(23) Fischer, C. F.; Idrees, M. Spline methods for resonances in photoionisation cross sections. J. Phys. B: At. Mol. Opt. Phys. 1990, 23, 679-691.

(24) Arneberg, R.; Müller, J.; Manne, R. Configuration interaction calculations of satellite structure in photoelectron spectra of $\mathrm{H}_{2} \mathrm{O}$. Chem. Phys. 1982, 64, 249-258.

(25) Szabo, A.; Ostlund, N. S. Modern Quantum Chemistry: Introduction to Advanced Electronic Structure Theory; Dover Publications: Mineola, NY, 1996.

(26) Bawagan, A. D.; Davidson, E. R. Understanding electron correlation: Recent progress in molecular synchrotron photoelectron spectroscopy. Adv. Chem. Phys. 1999, 110, 215-263.

(27) Schirmer, J. Beyond the random-phase approximation: A new approximation scheme for the polarization propagator. Phys. Rev. A 1982, 26, 2395-2416.

(28) Nesbet, R. Variational Methods in Electron-Atom Scattering Theory; Plenum Press: New York, 1980.

(29) Ortiz, J. V. Toward an Exact One-Electron Picture of Chemical Bonding. In Advances in Quantum Chemistry; Löwdin, P.-O., Ed.; Academic Press, 1999; Vol. 35, pp 33-52.

(30) Ortiz, J. V. Dyson-orbital concepts for description of electrons in molecules. J. Chem. Phys. 2020, 153, No. 070902.

(31) Ponzi, A.; Angeli, C.; Cimiraglia, R.; Coriani, S.; Decleva, P. Dynamical photoionization observables of the CS molecule: The role of electron correlation. J. Chem. Phys. 2014, 140, No. 204304.

(32) Ponzi, A.; Sapunar, M.; Angeli, C.; Cimiraglia, R.; Došlić, N.; Decleva, P. Photoionization of furan from the ground and excited electronic states. J. Chem. Phys. 2016, 144, No. 084307.

(33) Marante, C.; Klinker, M.; Corral, I.; González-Vázquez, J.; Argenti, L.; Martín, F. Hybrid-Basis Close-Coupling Interface to Quantum Chemistry Packages for the Treatment of Ionization Problems. J. Chem. Theory Comput. 2017, 13, 499-514.

(34) Bray, I.; Stelbovics, A. T. Convergent close-coupling calculations of electron-hydrogen scattering. Phys. Rev. A 1992, 46, 6995-7011.

(35) Carette, T.; Dahlström, J. M.; Argenti, L.; Lindroth, E. Multiconfigurational Hartree-Fock close-coupling ansatz: Application to the argon photoionization cross section and delays. Phys. Rev. A 2013, 87, No. 023420.

(36) Khatri, I.; Goyal, A.; Singh, A.; Singh, N.; Mohan, M. Photoionization of Cl-like Ni XII using relativistic R-matrix closecoupling method. Can. J. Phys. 2017, 95, 1136-1141.

(37) Toffoli, D.; Decleva, P. A Multichannel Least-Squares B-Spline Approach to Molecular Photoionization: Theory, Implementation, and Applications within the Configuration-Interaction Singles Approximation. J. Chem. Theory Comput. 2016, 12, 4996-5008.

(38) Bachau, H.; Cormier, E.; Decleva, P.; Hansen, J. E.; Martín, F. Applications of B-splines in atomic and molecular physics. Rep. Prog. Phys 2001, 64, 1815-1943.

(39) Majety, V. P.; Zielinski, A.; Scrinzi, A. Photoionization of few electron systems: a hybrid coupled channels approach. New J. Phys. 2015, 17, No. 063002.

(40) Tennyson, J. Electron-molecule collision calculations using the R-matrix method. Phys. Rep. 2010, 491, 29-76.

(41) Martin, R. L.; Shirley, D. A. Theory of core-level photoemission correlation state spectra. J. Chem. Phys. 1976, 64, 3685-3689.

(42) Toffoli, D.; Stener, M.; Fronzoni, G.; Decleva, P. Convergence of the multicenter B-spline DFT approach for the continuum. Chem. Phys. 2002, 276, 25-43.

(43) Stener, M.; Furlan, S.; Decleva, P. Density functional calculations of photoionization with an exchange-correlation potential with the correct asymptotic behaviour. J. Phys. B: At., Mol. Opt. Phys. 2000, 33, $1081-1102$.

(44) Nakano, M.; Selles, P.; Lablanquie, P.; Hikosaka, Y.; Penent, F.; Shigemasa, E.; Ito, K.; Carniato, S. Near-Edge X-Ray Absorption Fine Structures Revealed in Core Ionization Photoelectron Spectroscopy. Phys. Rev. Lett. 2013, 111, No. 123001.

(45) Ponzi, A.; Quadri, N.; Angeli, C.; Decleva, P. Electron correlation effects in the photoionization of $\mathrm{CO}$ and isoelectronic diatomic molecules. Phys. Chem. Chem. Phys. 2019, 21, 1937-1951.

(46) Casida, M. E. Time-dependent density-functional theory for molecules and molecular solids. J. Mol. Struct.: THEOCHEM 2009, 914, 3-18.

(47) Zangwill, A.; Soven, P. Density-functional approach to local-field effects in finite systems: Photoabsorption in the rare gases. Phys. Rev. A 1980, 21, 1561-1572.

(48) Thouless, D. The Quantum Mechanics of Many-Body Systems, 2nd ed.; Dover Books on Physics; Dover Publications, 2013.

(49) Mahan, G. D.; Subbaswamy, K. Local Density Theory of Polarizability; Plenum Press: New York, 1990.

(50) Stener, M.; Fronzoni, G.; Decleva, P. Time-dependent densityfunctional theory for molecular photoionization with noniterative algorithm and multicenter B-spline basis set: $\mathrm{CS}_{2}$ and $\mathrm{C}_{6} \mathrm{H}_{6}$ case studies. J. Chem. Phys. 2005, 122, No. 234301.

(51) de Boor, C. A Practical Guide to Splines; Springer: New York, 1978.

(52) Koopmans, T. Über die Zuordnung von Wellenfunktionen und Eigenwerten zu den Einzelnen Elektronen Eines Atoms. Physica 1934, $1,104-113$

(53) Stanton, J. F.; Bartlett, R. J. The equation of motion coupledcluster method. A systematic biorthogonal approach to molecular excitation energies, transition probabilities, and excited state properties. J. Chem. Phys. 1993, 98, 7029-7039.

(54) Bartlett, R. J. Coupled-cluster theory and its equation-of-motion extensions. WIREs Comput. Mol. Sci. 2012, 2, 126-138.

(55) Helgaker, T.; Jørgensen, P.; Olsen, J. Molecular ElectronicStructure Theory; John Wiley \& Sons: Chichester, 2000.

(56) Vidal, M. L.; Krylov, A. I.; Coriani, S. Dyson orbitals within the fcCVS-EOM-CCSD framework: theory and application to X-ray photoelectron spectroscopy of ground and excited states. Phys. Chem. Chem. Phys. 2020, 22, 2693-2703.

(57) Vidal, M. L.; Krylov, A. I.; Coriani, S. Correction: Dyson orbitals within the fc-CVS-EOM-CCSD framework: theory and application to $\mathrm{X}$-ray photoelectron spectroscopy of ground and excited states. Phys. Chem. Chem. Phys. 2020, 22, 3744-3747.

(58) Chandra, N. Photoelectron spectroscopic studies of polyatomic molecules. I. Theory. J. Phys. B: At. Mol. Phys. 1987, 20, 3405-3415.

(59) Stener, M. Photoionization of oriented molecules: a time dependent density functional approach. Chem. Phys. Lett. 2002, 356, $153-160$.

(60) NIST Computational Chemistry Comparison and Benchmark Database, NIST Standard Reference Database Number 101. Release 20 August 2019

(61) Moncrieff, D.; Hillier, I. H.; Saunders, V. R.; von Niessen, W. Correlation effects in the ground and ionic states of $\operatorname{bis}(\pi$-allyl $)$ nickel. Inorg. Chem. 1985, 24, 4247-4251.

(62) te Velde, G.; Bickelhaupt, F. M.; Baerends, E. J.; Fonseca Guerra, C.; van Gisbergen, S. J. A.; Snijders, J. G.; Ziegler, T. Chemistry with ADF. J. Comput. Chem. 2001, 22, 931-967.

(63) Van Lenthe, E.; Baerends, E. J. Optimized Slater-type basis sets for the elements 1-118. J. Comput. Chem. 2003, 24, 1142-1156.

(64) Shao, Y.; Gan, Z.; Epifanovsky, E.; Gilbert, A. T.; Wormit, M.; Kussmann, J.; Lange, A. W.; Behn, A.; Deng, J.; Feng, X.; Ghosh, D.; Goldey, M.; Horn, P. R.; Jacobson, L. D.; Kaliman, I.; Khaliullin, R. Z.; Kuś, T.; Landau, A.; Liu, J.; Proynov, E. I.; Rhee, Y. M.; Richard, R. M.; Rohrdanz, M. A.; Steele, R. P.; Sundstrom, E. J.; H, L. W., III; Zimmerman, P. M.; Zuev, D.; Albrecht, B.; Alguire, E.; Austin, B.; Beran, G. J. O.; Bernard, Y. A.; Berquist, E.; Brandhorst, K.; Bravaya, K. B.; Brown, S. T.; Casanova, D.; Chang, C.-M.; Chen, Y.; Chien, S. H.; Closser, K. D.; Crittenden, D. L.; Diedenhofen, M.; R, A. D., Jr.; Do, H.; 
Dutoi, A. D.; Edgar, R. G.; Fatehi, S.; Fusti-Molnar, L.; Ghysels, A.; Golubeva-Zadorozhnaya, A.; Gomes, J.; Hanson-Heine, M. W.; Harbach, P. H.; Hauser, A. W.; Hohenstein, E. G.; Holden, Z. C.; Jagau, T.-C.; Ji, H.; Kaduk, B.; Khistyaev, K.; Kim, J.; Kim, J.; King, R. A.; Klunzinger, P.; Kosenkov, D.; Kowalczyk, T.; Krauter, C. M.; Lao, K. U.; Laurent, A. D.; Lawler, K. V.; Levchenko, S. V.; Lin, C. Y.; Liu, F.; Livshits, E.; Lochan, R. C.; Luenser, A.; Manohar, P.; Manzer, S. F.; Mao, S.-P.; Mardirossian, N.; Marenich, A. V.; Maurer, S. A.; Mayhall, N. J.; Neuscamman, E.; Oana, C. M.; Olivares-Amaya, R.; O’Neill, D. P.; Parkhill, J. A.; Perrine, T. M.; Peverati, R.; Prociuk, A.; Rehn, D. R.; Rosta, E.; Russ, N. J.; Sharada, S. M.; Sharma, S.; Small, D. W.; Sodt, A.; Stein, T.; Stück, D.; Su, Y.-C.; Thom, A. J.; Tsuchimochi, T.; Vanovschi, V.; Vogt, L.; Vydrov, O.; Wang, T.; Watson, M. A.; Wenzel, J.; White, A.; Williams, C. F.; Yang, J.; Yeganeh, S.; Yost, S. R.; You, Z.-Q.; Zhang, I. Y.; Zhang, X.; Zhao, Y.; et al. Advances in molecular quantum chemistry contained in the Q-Chem 4 program package. Mol. Phys. 2015, 113, 184-215.

(65) Baltzer, P.; Karlsson, L.; Lundqvist, M.; Wannberg, B.; Holland, D.; MacDonald, M. An experimental study of the valence shell photoelectron spectrum of hydrogen sulphide. Chem. Phys. 1995, 195, 403-422.

(66) Dehmer, J. L.; Dill, D.; Parr, A. C. Photoionization Dynamics of Small Molecules. In Photophysics and Photochemistry in the Vacuum Ultraviolet; McGlynn, S. P.; Findley, G. L.; Huebner, R. H., Eds.; Springer Netherlands: Dordrecht, 1985; pp 341-408.

(67) Green, J. C.; Decleva, P. Photoionization cross-sections: a guide to electronic structure. Coord. Chem. Rev. 2005, 249, 209-228. Synchrotron Radiation in Inorganic and Bioinorganic Chemistry.

(68) Adam, M. Y.; Naves de Brito, A.; Keane, M. P.; Svensson, S.; Karlsson, L.; Källne, E.; Correia, N. The high resolution inner-valence photoelectron spectrum of $\mathrm{H}_{2} \mathrm{~S}$ induced by monochromatic $\mathrm{Al} \mathrm{K}_{\alpha} \mathrm{X}$ rays. J. Electron Spectrosc. Relat. Phenom. 1991, 56, 241-257.

(69) Carlson, T. A.; Krause, M. O.; Grimm, F. A. Angle resolved photoelectron spectroscopy of $\mathrm{CS}_{2}$ and COS measured as a function of photon energy from 21 to $70 \mathrm{eV}$. J. Chem. Phys. 1982, 77, 1701-1709.

(70) Carlson, T. A.; Krause, M. O.; Grimm, F. A.; Allen, J. D.; Mehaffy, D.; Keller, P. R.; Taylor, J. W. Angle-resolved photoelectron spectroscopy of $\mathrm{CS}_{2}$ and $\mathrm{COS}$ measured with synchrotron radiation. J. Chem. Phys. 1981, 75, 3288-3292.

(71) Carnovale, F.; White, M.; Brion, C. Absolute dipole oscillator strengths for photoabsorption and photoionization of carbon disulphide. J. Electron Spectrosc. Relat. Phenom. 1981, 24, 63-76.

(72) Christiansen, O.; Koch, H.; Jørgensen, P. Perturbative triple excitation corrections to coupled cluster singles and doubles excitation energies. J. Chem. Phys. 1996, 105, 1451-1459.

(73) Rohmer, M. M.; Demuynck, J.; Veillard, A. A “double-zeta” type wavefunction for an organometallic: Bis- $(\pi$-allyl)nickel. Theoret. Chim. Acta 1974, 36, 93-102.

(74) Batich, C. D. Photoelectron spectroscopy of bis $(\pi$-allyl) nickel and its methyl substituted derivatives: support for the near validity of Koopmans' theorem. J. Am. Chem. Soc. 1976, 98, 7585-7590.

(75) Li, X.; Bancroft, G. M.; Puddephatt, R. J.; Hu, Y. F.; Liu, Z.; Tan, K. H. Reassignment of the valence photoelectron spectra of nickel diallyl complex $\mathrm{Ni}\left(\eta^{3}-\mathrm{C}_{3} \mathrm{H}_{5}\right)_{2}$ using variable energy photoelectron spectroscopy. Inorg. Chem. 1992, 31, 5162-5163.

(76) Li, X.; Bancroft, G. M.; Puddephatt, R. J.; Liu, Z. F.; Hu, Y. F.; Tan, K. H. Variable Energy Photoelectron Spectroscopy of $\mathrm{M}\left(\eta^{3}\right.$ $\left.\mathrm{C}_{3} \mathrm{H}_{5}\right)_{2}(\mathrm{M}=\mathrm{Ni}, \mathrm{Pd}$, and $\mathrm{Pt})$ : Molecular Orbital Assignments. J. Am. Chem. Soc. 1994, 116, 9543-9554.

(77) Von Niessen, W.; Bieri, G.; Schirmer, J.; Cederbaum, L. S. Holemixing effects in the ionization of some unsaturated oxo-compounds. Chem. Phys. 1982, 65, 157-176.

(78) Decleva, P.; Fronzoni, G.; Stener, M. Giant correlation effects in the photoelectron spectrum of $\mathrm{Ni}\left(\mathrm{C}_{3} \mathrm{H}_{5}\right)_{2}$ : clues from accurate calculation of ionization cross-sections. Theor. Chem. Acc. 2012, 131, No. 1185. 